Western University Scholarship@Western

2018

\title{
Microencapsulation by in situ polymerization of amino resins
}

\author{
Olivier Nguon \\ Western University \\ Francois Lagugne-Labarthet \\ Western University \\ 3M Company Canada \\ Elizabeth R. Gillies \\ Western University, egillie@uwo.ca
}

Follow this and additional works at: https://ir.lib.uwo.ca/chempub

\section{Part of the Chemistry Commons}

\section{Citation of this paper:}

Nguon, Olivier; Lagugne-Labarthet, Francois; 3M Company Canada; and Gillies, Elizabeth R., "Microencapsulation by in situ polymerization of amino resins" (2018). Chemistry Publications. 110.

https://ir.lib.uwo.ca/chempub/110 


\title{
Microencapsulation by in situ polymerization of amino resins
}

Olivier Nguon, ${ }^{a, b}$ François Lagugné-Labarthet, ${ }^{a}$ Frank Brandys, ${ }^{b} \mathrm{Jian} \mathrm{Li},{ }^{b} *$ and Elizabeth R.

$$
\text { Gillies, }{ }^{a, c, *}
$$

${ }^{a}$ Department of Chemistry, The University of Western Ontario, 1151 Richmond Street, London,

Ontario, Canada N6A 5B7

${ }^{b} 3 M$ Canada Company, 1840 Oxford Street East, London, Ontario, Canada N5V 3R6

${ }^{c}$ Department of Chemical and Biochemical Engineering, The University of Western Ontario, 1151 Richmond Street, London, Ontario, Canada N6A $5 B 9$

*Corresponding authors: Elizabeth R. Gillies (egillie@uwo.ca), Jian Li (jli35@mmm.com)

Keywords: microcapsule, encapsulation, in situ polymerization, urea-formaldehyde, amino resin, mechanism

Title running head: Microencapsulation using amino resins

\begin{abstract}
By surrounding small droplets with a coating, one can obtain micrometer-size capsules (microcapsules), and combine multiple properties into a single system. This technology has allowed the design of advanced and functional materials. Amino resins are composed principally of urea and/or melamine and formaldehyde, and exhibit advantages as wall-forming materials, such as high mechanical strength and chemical resistance. In this review, a general description of the encapsulation process by in situ polymerization of amino resins is given. Characterization methods, and the influence of the physical and design parameters are discussed. A mechanistic description, and some of the promising avenues of research are also presented.
\end{abstract}




\section{Introduction}

The surrounding of a core* component by a wall ${ }^{\phi}$ material offers the possibility to combine the properties of multiple constituents into a single system. At the microscale level, the spherical construction thus formed is known as a microcapsule (MC). The process of encapsulation has been extensively employed in nature in numerous forms (e.g., cells, seeds, eggs), but microencapsulation came to prominence for material scientists after the seminal work by Green in the mid-1950s. ${ }^{1-3}$ The encapsulation process has since been described in the patent literature, in reviews, ${ }^{4-17}$ and in books. ${ }^{18-21}$ This versatile technology enables the segregation of a core material from its environment for preservation, safety, controlled release, or the enhanced processing, mixing and handling of a material. ${ }^{21}$ This process has been exploited in a variety of industries ranging from pharmaceuticals, cosmetics, food additives, pesticides, to industrial chemicals and adhesives. ${ }^{14}$ In recent years, microencapsulation has gained new interest thanks in part to the numerous applications that can be envisioned. For instance, the controlled release of a core material enables the formulation of versatile adhesives or drugs with enhanced or even unique properties. Currently, the design of smart or multi-functional materials from MCs represents a promising field for both academic research and industries. ${ }^{5}$

Since the first application of microencapsulation technology to the production of carbonless copy paper, ${ }^{1}$ many synthetic protocols (well over $200^{22,23}$ ) and applications have been reported. A broad range of core materials has been encapsulated using various wall materials such as organic polymers, fats, waxes, ${ }^{22,24}$ and more recently inorganic compounds. ${ }^{25}$

MCs composed of urea and/or melamine and formaldehyde (Figure 1) as wall-forming materials, often termed amino resins or aminoplasts, are of particular interest due to their excellent properties including high mechanical strength, ${ }^{26}$ high loading, good thermal stability, ${ }^{27}$ water ${ }^{28}$ and chemical resistance, ${ }^{29,30}$ long term storage stability, ${ }^{30}$ low toxicity of the cured resin, ${ }^{31}$ low 
permeability, ${ }^{32}$ low cost, and the propensity for industrial scale-up. ${ }^{31}$ However, a precise control over the microencapsulation process is critical to the rational design and performance of MCs in targeted applications. Although many reports exist for the encapsulation of various core materials, few recent articles have reviewed the preparation and application of amino resins..$^{23,33-35}$ The complexities of the process render material design challenging, and it has often remained an empirical endeavor. However, renewed interest in the field has shed light on some of the mechanistic details of microencapsulation by in situ polymerization.

After introducing the major types of encapsulation processes (coacervation, interfacial polymerization, and in situ polymerization), the chemistry of amino resins is described. The utilization of amino resin as wall-forming materials for microencapsulation is then covered in detail. Particular attention is placed on the influence of the processing parameters. A more general mechanistic description of the encapsulation technology then follows, before concluding the review with a discussion of some of the promising opportunities for research.

\subsection{Microencapsulation techniques}

Synthetic strategies for microencapsulation can be broadly divided among two categories, namely chemical (or type A) and physical/mechanical (or type B) methods ${ }^{\varphi}$ (Table 1). ${ }^{10,12,36}$ Processes in the first category rely solely on wet chemistry protocols for capsule formation, and proceed from the reaction of monomers, oligomers, or preformed polymeric species as starting materials. As illustrated in Figure 2, chemical microencapsulation methods generally involve the initial dispersion or emulsion of the core material, followed by capsule-wall deposition, and ultimately recovery of the MCs. ${ }^{37}$ Among such methods, important processes include complex coacervation, interfacial polymerization, and in situ polymerization. Physical/mechanical 
processes on the other hand typically involve a gas phase medium during deposition or spraying of a coating material. ${ }^{12}$ The latter techniques, including spray-drying, ${ }^{38,39}$ have been historically important in foraying current microencapsulation technology and have been presented in detail elsewhere. $^{36}$

\subsection{Chemical processes}

\subsubsection{Complex coacervation}

Chemical processes have proven to be versatile methods in the design of multi-functional materials, and in the study of the mechanism of encapsulation. ${ }^{10}$ In the process of coacervation (from the Latin acervus, heap), partial desolvation of a polymer solution yields two liquid phases, one rich and one poor in polymer (Figure $3 a) .{ }^{40}$ Desolvation can be induced by addition of a salt, ${ }^{3}$ precipitant, non-solvent, or polymer, as well as by a change in $\mathrm{pH}^{3}$ or temperature. ${ }^{9}$ Alternatively, coacervation can occur by precipitation of oppositely charged polymers in a process known as complex coacervation. ${ }^{3}$ In the presence of a water-insoluble core material, the coacervate phase deposits as a thin film around the dispersed phase, and capsule formation occurs upon hardening of the polymeric film. ${ }^{41}$ Coacervation processes have been widely applied to the formation of MCs; however, they exhibit some limitations. The process is dependent on the concentrations of the polymer and the electrolyte. The $\mathrm{pH}$ must be carefully adjusted during complex coacervation, particularly when employing polymers with isoelectric points. ${ }^{42}$ Natural products are often employed for the capsule formation, but wall permeability, degradation and relatively high costs can mare the process. ${ }^{43}$ 


\subsubsection{Interfacial polymerization}

Interfacial polymerization is another well-studied encapsulation technique. In this process, reactive monomers or prepolymers are dissolved in two immiscible phases (Figure 3b). Upon droplet formation by dispersion, polymerization takes place at/on the interface and results in MC formation. ${ }^{44}$ This technique has been used to obtain relatively small capsules (3-6 $\left.\mu \mathrm{m}\right)$, and has been employed in a variety of applications (energy storage, pharmaceutics, cosmetics, agriculture, etc.). One disadvantage of the technique is that the formation of a thin interfacial polymeric layer between the reagents can hinder further reaction. Capsules with low mechanical integrity may then be produced. ${ }^{43}$ The presence of a reactive monomer in the core phase can also be detrimental to the encapsulated species. ${ }^{41}$ Additionally, diffusion of monomers into the core phase can promote the formation of solid microspheres rather than MCs. ${ }^{6}$

\subsubsection{In situ polymerization}

Several encapsulation processes rely on the in situ polymerization technique, and have been discussed briefly in several reviews. ${ }^{6,11,23,33-35,41,45}$ In such processes, a solution of the monomeric or oligomeric wall material is added to the core phase, the latter being dispersed to the desired size (Figure 3c). Controlled deposition and precipitation of the polymer takes place at the interface by using precipitants, or a change in $\mathrm{pH}$, temperature, or solvent quality.

Arshady and George distinguished three cases of in situ polymerization based on the solubility of the monomer and the polymer. ${ }^{6}$ Suspension polymerization takes place when the monomer is insoluble in the dispersion medium and forms suspended monomer droplets that polymerize in solution to yield polymer microparticles. The polymerization reactor and stirring rate are thus important parameters in maintaining a uniform size distribution. In another case, 
precipitation polycondensation takes place when the monomer but not the polymer is soluble in the dispersion medium. As the reaction proceeds, flocculation and aggregation of a (low molar mass) polymer yield particles with a characteristically broad size distribution and irregular shape. Lastly, dispersion polycondensation takes place when the dispersion medium is a good solvent for the monomer, but a poor solvent for the polymer. Under such conditions, swelling of the polymer rather takes place and microcapsule growth occurs by the sustained addition of monomer and oligomer to the particle. Microparticles with a narrow size distribution are formed under these conditions.

\subsection{Amino resin microcapsules}

The use of amino resins prepared from urea and/or melamine and formaldehyde constitutes the majority of the applications regarded as in situ polymerization. An early patent by Veatch and Burhans, in 1957, demonstrated the utilization of resins of phenol, formaldehyde, and urea or melamine for the preparation of hollow MCs using a spray-drying technique. ${ }^{46}$ Subsequent patents by Macaulay ${ }^{39}$ and Soloway ${ }^{47}$ described a solution-based approach for encapsulating carbon black and natural liquid products, respectively, using urea-formaldehyde (UF) resins and surfactants. Impregnated particulates with biologically active compounds were also encapsulated with amino resins by Geary. ${ }^{48}$

The first commercially important procedure was patented by Matson for 3M Corporation. The inventor disclosed the large-scale preparation of UF MCs with superior properties, such as toughness and impermeability. ${ }^{49}$ The procedure involved the formation of a precondensate (or prepolymer) composed of UF oligomers without the need for surfactants. The encapsulation of a variety of core materials was reported, including solids (sulfur), gases (air, vaporized organic 
solvents), and liquids. In the latter category in particular, a wide variety of suitable oily liquid cores was presented including fatty acids, solvents, dyes, polyacids, polysulfides, perfumes, agricultural chemicals, biological products, pharmaceuticals, adhesives, light-sensitive materials, photographic materials, cleaners, monomers and polymerization initiators. Interestingly, the properties of the polymeric wall could be modified by including co-reactants (modifiers) to the prepolymer solution such as guanidine hydrochloride, thiourea, phenol, hydrazine, and melamine. The capsule toughness and permeability were also controlled by adding a salt or by adjusting the $\mathrm{pH}$ during the synthesis. The formulation of the capsules onto sheets for cleaning, copying, printing or paper coating was proposed. ${ }^{50}$ For instance, electrically rupturable capsules were obtained by deposition of a conductive layer onto the MCs, and used in copy and light sensitive sheets or to trigger the release and reaction of various chemicals. ${ }^{51}$

Since the reports by Matson et al., amino resin MCs have attracted great commercial interest, particularly for the design of pressure-sensitive recording and adhesive materials, ${ }^{52}$ agrochemicals, perfumes, and vegetables oils. Dietrich et al. tabulated at least 205 patents filed among 20 companies by $1989 .{ }^{35}$ More recently, as compiled by Duan, applications of interest include consumer products, flame retardants, phase change materials, electronic inks, thermalsensitive paper, self-healing agents, ${ }^{53}$ drag-reducing agents, smart coatings,${ }^{54}$ and polymer additives. ${ }^{33}$ The renewed academic and industrial interest in recent years can be illustrated by the growing number of publications and patents in the area of amino resin microencapsulation, as shown in Figure 4.

Figure 5 illustrates the general process used for the preparation of MCs by in situ polymerization, and is based on the encapsulation of epoxy resins as a representative example. ${ }^{30,55-}$ ${ }^{58}$ Typically, the process starts from an aqueous precondensate (prepolymer) solution of urea and 
formaldehyde, with triethanolamine used to adjust the $\mathrm{pH}$ to 8-9. Modifiers are also added. For instance, ammonium chloride is used as a hardener, and resorcinol promotes branching formation and increases water resistance. ${ }^{58}$ The $\mathrm{pH}$ is then adjusted prior to the addition of the core material (e.g., epoxy resin, fragrance, pharmaceutical). Emulsification of the oil and water phases is achieved using mechanical stirring. It should be noted that the encapsulation process can also start from the dissolution of the amine monomer alone (e.g., urea), formaldehyde being added only after the emulsification stage. ${ }^{5-60}$ In situ polymerization is initiated by increasing the temperature, and/or adjusting the $\mathrm{pH}$ of the solution. At the end of the reaction, the solution is then neutralized. Scavengers can also be added to remove the unreacted formaldehyde. Recovery of the capsules is easily achieved by filtration, followed by several washes, and drying of the MCs.

\section{Amino resins}

\subsection{Polycondensation reactions}

Amino resin-based MCs have been predominantly prepared from the reaction of urea and/or melamine with formaldehyde. ${ }^{23,35}$ As described above, the capsule synthesis is often a twostep process that first proceeds by the preparation of an amino prepolymer solution. Further condensation of the amino resin leads to the formation of a polymeric network that forms at the surface of the dispersed phase. The polycondensation reactions taking place are complex, owing to the multi-functionality of the reactants. Even though numerous reports have investigated the synthesis and properties of amino resins, ${ }^{61-86}$ a complete understanding of the molecular mechanisms involved remains elusive. ${ }^{87}$ 


\subsection{Formaldehyde solution}

In aqueous solution, formaldehyde is readily hydrated to methylene glycol, and only a trace amount of non-hydrated formaldehyde is present (typically less than 0.1 mol \%). From measurements of the absorption rate of formaldehyde in water, Winkelman et al. determined the chemical equilibrium constant of hydration to be in the form $K_{h}=\mathrm{e}^{3769 / T-5.494}$, where $T$ is the temperature in Kelvin (Scheme 1). ${ }^{88}$ Further reaction of methylene glycol in water yields poly(methylene glycol)s. Commercial solutions frequently contain 30-55 mass percent (wt \%) formaldehyde in water (formalin), and are acidic with a $\mathrm{pH}$ ranging from 2.5 to $4.5{ }^{89,90} \mathrm{~A}$ progressive increase in the acidity occurs via formation of traces amount of formic acid according to the Cannizarro reaction $\left(2 \mathrm{CH}_{2} \mathrm{O}+\mathrm{H}_{2} \mathrm{O} \rightleftarrows \mathrm{HCOOH}+\mathrm{CH}_{3} \mathrm{OH}\right),{ }^{91,92}$ and/or the presence of methyl formate. ${ }^{93}$

The addition of an alcohol, such as methanol, inhibits the polymerization of formaldehyde by shifting the equilibrium towards monomeric or low-molar-mass oligomeric species, and promotes the formation of alkoxylated compounds, primarily hemiacetals (Scheme 1 and 2). ${ }^{90}$ In fact, methanol is typically added as a stabilizer to formaldehyde solutions to prevent the formation of polymeric species whose solubility decreases when the degree of polymerization becomes greater than three. ${ }^{89}$ In a detailed study of the equilibrium speciation by nuclear magnetic resonance (NMR) spectroscopy in aqueous methanol-formaldehyde solutions, Gaca et al. formulated a quantitative equilibrium model for the formation of methylene glycol dimers and trimers and the corresponding methoxylated products (Scheme 2). ${ }^{90}$ They confirmed the shift of the equilibrium upon dilution towards the formation of monomeric species, and the nondependence of the equilibrium of formation of di(methylene glycol) on the temperature. Ott et al. calculated the rate constants of degradation of poly(methylene glycol)s in water or methanol and 
revealed a correlation between dilution, higher temperatures and acidic or basic conditions and the formation of methylene glycol. ${ }^{94}$

\subsection{Reaction of urea and formaldehyde}

In the preparation of a polymeric network from formaldehyde and urea/melamine, four stages have been identified (Scheme 3). ${ }^{62,87}$ In the first stage, condensation reactions between formaldehyde and the amino-containing compound yield hydroxymethylated products (methylolation). The multi-functionality of urea allows for the substitution reactions to take place at up to three of the amide protons (complete methylolation having not been reported). The reaction follows a general acid-base catalysis scheme, and is typically performed at a $\mathrm{pH}$ ranging from 7 to 9. ${ }^{62,95,96}$ In a second stage, further reaction between the methylol ureas and methylene glycol/formaldehyde form oligomeric hemiformals. This reaction is analogous to the formation of poly(methylene glycol) in aqueous formaldehyde solution, and is similarly hindered by the presence of alcohols. In the third stage, lowering of the $\mathrm{pH}$ promotes condensation reactions between the intermediate species and yields methylene-bridged and ether-bridged compounds. (One can note that UF resins are frequently synthesized under acidic conditions, while melamine containing resins are rather prepared under a mildly alkaline $\mathrm{pH} .{ }^{97}$ ) Given the multi-functionality of the oligomers present, a thermoset network is then assembled in the last stage of the process.

The rates of the reactions taking place depend on the concentration of acid or base, and one can control the advancement of each stage by adjusting the $\mathrm{pH}$ during the process. The addition reaction of urea with formaldehyde is catalyzed both by acids and bases, while the condensation of methylol ureas with urea is predominantly acid-catalyzed (Figure 6). 
While many studies have investigated the species formed during the reaction of urea and formaldehyde, few have studied the mechanism of formation of the polymeric network. Glutz and Zollinger demonstrated that the kinetics of the reaction between urea and formaldehyde follows an acid/base catalyzed mechanism. ${ }^{98}$ The rate constants were found to exhibit minima at $\mathrm{pH}$ values ranging from 5 to 8 . Participation of the dehydrated form of formaldehyde rather than methylene glycol was proposed ${ }^{68}$ while the formation of an intermediate carbonium ion during the reaction was suggested by several authors. ${ }^{65,69,74,96}$

From computational studies, $\mathrm{Li}$ et al. investigated the mechanism of the acid-catalyzed reaction of urea and formaldehyde. ${ }^{99}$ The overall reaction pathway is shown in Scheme 4. The authors accounted for the slow reaction between formaldehyde and urea under neutral conditions, and argued that a strong $\mathrm{p}-\pi$ conjugation between the amino and the carbonyl groups in urea reduced the nucleophilic character of the molecule. Under acidic conditions, however, protonation of formaldehyde or methylene glycol catalyzed the reaction with urea according to an $\mathrm{S}_{\mathrm{N}} 2$ mechanism and resulted in the formation of $N$-protonated methylol urea. Proton transfer to the oxygen then proceeded via an intramolecular or a water-catalyzed mechanism. Dehydration of the protonated compound occurred at a relatively low energy cost (particularly via an intramolecular proton-transfer pathway) and yielded a stable methylol carbonium. This compound readily reacted with urea and formed a methylene-bridged diurea cation. Similarly, the reaction of $O$-protonated methyl urea with urea was determined to be exothermic and yielded diurea species. The eventual deprotonation of the methylene-bridge diurea cation in water occurred via a barrierless process when more than three water molecules were included in the calculation. Reaction of the $O$ protonated methyl urea or methylol carbonium with methylol urea rather formed ether-bridged diurea species. These compounds were found to be less stable under acidic conditions however. It 
is noteworthy that in the acid-catalyzed reaction of urea and formaldehyde, protonated urea was deemed non-reactive towards formaldehyde, and thought rather to hinder the reaction.

The effect of the $\mathrm{pH}$ on the reaction rate was confirmed experimentally by Nair and Francis who reported the predominant formation of methylene-bridged compounds under acidic conditions, while methylol ureas formed at a neutral or high $\mathrm{pH} .{ }^{96} \mathrm{~A}$ similar observation was made on insoluble resins using solid state NMR spectroscopy. ${ }^{100}$ The addition of formaldehyde to urea was found to be increasingly difficult as the degree of substitution of the latter increased. The rate of methylolation was lowered by about a factor of three after each substitution reaction. ${ }^{65,73}$ Similarly, further condensation reactions between urea and methylol ureas took place at a slower rate when the degree of methylolation increased. ${ }^{96}$ The electron-withdrawing property of the methylol groups was suggested to be detrimental to substitutions, and to result in mutual deactivation in dimethylol urea. The growth of the polymeric chains continued predominantly by further addition of urea and formaldehyde molecules rather than condensation between oligomeric chains, which was attributed to the low reactivity of amide nitrogens. ${ }^{96}$ Lastly, one should note that all the reactions are reversible, so that dissociation to urea and formaldehyde can potentially take place.

\subsection{Colloidal properties}

As described above, the complex series of reactions taking place during the preparation of amino resins yields a mixture of products with evolving properties. The formation of UF, melamine-formaldehyde (MF), and melamine-urea-formaldehyde (MUF) resins proceeds from soluble reagents that organize into an intermediate colloidal sol prior to gelation and precipitation

(Figure 7a). ${ }^{101,102}$ In the first stage of the polymerization reaction between urea and formaldehyde, 
a reduction in the number of hydrogen-bonding units on the oligomeric chains may induce aggregate formation. ${ }^{103}$ From size exclusion chromatography analysis, the solubility limit for the linear oligomers occurred when urea-terminated species with 4-8 urea units were formed. ${ }^{104}$ Interestingly, the molecular aggregation appears to be driven mainly by changes in terms of chemical composition (-OH group content), rather than molecular size. ${ }^{101}$ The heterogeneous solution, therefore, contains a mixture of soluble oligomers and reagents, and swollen molecular aggregates. Imaging by electron microscopy indicated that the shape of sub-micron aggregates is dependent on the resin composition. ${ }^{102}$ Lamellar morphologies were observed with UF resins. Indeed, in analogy to polypeptides, the formation of helical ( $\pi$-helix) and planar ( $\alpha$-sheet) structures during curing has been postulated. ${ }^{103}$ Globular aggregates were rather formed in MF resins, and an intermediate morphology was observed for MUF resins displaying short rod-like structures.

Several mechanisms have been proposed to account for the colloidal stability of the aggregates. The formation of a solvation layer by unreacted urea, hindering hydrogen bonding between aggregates, may contribute to the stabilization. ${ }^{101}$ Electrostatic stabilization has also been postulated to occur through the formation of an ionic double layer composed of hydronium ions and formaldehyde molecules. ${ }^{105}$ Ferra et al. discussed the participation of polymeric species with amphiphilic character distributed at the surface of the dispersed phase. ${ }^{101}$ Hydrophilic end-groups (e.g., hydroxymethylene, monofunctionalized urea) on the polymer chains can interact with ionic species (e.g., $\mathrm{Na}^{+}$and $\mathrm{H}^{+}$) and generate electrostatic repulsions.

As the proposed stabilization mechanisms depend on the concentration of the charged species, flocculation of the aggregates can be induced upon dilution (Figure $7 \mathrm{~b}$ ). In fact, the size and extent of aggregate formation was found to depend on the level of condensation and ageing of 
the resin. Coalescence of particles in the aggregates yielded larger species, and resulted in a globular morphology prior to gelation. ${ }^{105}$ The viscosity during the polymerization reaction was found to increase over time, and Mehdiabadi et al. proposed the relationship given by Equation 1, to account for the change in the kinematic viscosity $v$ (in $\mathrm{cSt}$ ) as a function of the $\mathrm{pH}$ of the solution and time $(t) .{ }^{106}$

$$
\ln \left(\frac{v}{9.43}\right)=\left(0.001586+1337.938 \times 10^{-p H}\right) \times t
$$

\section{Control of the encapsulation process}

\subsection{Properties of microcapsules}

Control over the properties of MCs is essential for the rational design of materials for targeted applications. The physical and chemical properties of MCs are a result of the synthesis and processing parameters employed in their preparation. Properties of MCs such as the morphology, size and size distribution, wall thickness, encapsulation efficiency, loading level, release profile, and mechanical properties will determine their end-application. The nature of the molecular species in the amino resins and the presence of surfactants are other parameters of importance. By adjusting the $\mathrm{pH}$ and the temperature, one can also influence the type of reactive species involved. The nature of the core material, as well as the ratio of the core/shell materials are other essential variables. Furthermore, additives such as salts and formaldehyde scavengers have been found to influence the capsule formation. Process parameters such as the design of the reactor, and stirring rate have also been found to be critical in the preparation of MCs and will be discussed below. 


\subsection{Characterization of microcapsules}

A range of characterization techniques is available to probe the influence of the process parameters, and to monitor MC quality during formulation, storage, application, and disposal/recycling. Y. Zhang and Rochefort, ${ }^{44}$ and Z. Zhang and coworkers ${ }^{107}$ reviewed important methods used for the characterization of the physicochemical, structural, and mechanical properties of MCs. The techniques for the characterization of physicochemical and structural properties are summarized in Table 2, while those used for measuring the mechanical properties are summarized in Table 3. In practice, the choice of the characterization technique will be dictated by the end-use of the MCs, although interdependency between properties is to be expected. For example, the wall thickness will affect the mechanical strength of the MCs, but it will also influence the release rate of a core material in load-delivery applications.

The size and size distribution of MCs are readily obtained from laser diffraction measurements. ${ }^{55}$ For nanosized materials, dynamic light scattering (DLS) is preferred. ${ }^{108}$ Inprocess measurements find important applications in industrial settings, and often rely on laserbeam analyzers measuring the microcapsule's chord length (geometric line on the surface of a particle). ${ }^{109}$ Examples of such techniques include focused beam reflectance measurement, and spatial filtering velocity. More recently, photometric stereo imaging was used to obtain a 3D image and particle size distribution by using light pulses. ${ }^{110}$

Optical microscopy allows the rapid visualization of MCs, but the resolution is limited to about half of the minimum wavelength of visible light $(0.2 \mu \mathrm{m})$. Electron microscopy such as scanning electron microscopy $(\mathrm{SEM}),{ }^{111}$ environmental SEM (ESEM), ${ }^{112}$ and transmission electron microscopy (TEM), ${ }^{113}$ offer better resolution for the characterization of the surface roughness, and wall thickness. In combination with a focused ion beam (FIB) technique, an 
accurate cross section of the microcapsules can be obtained and provide further information on the microcapsule structure. ${ }^{114}$ Confocal laser scanning microscopy is another imaging technique with a resolution in the range of $0.2-0.5 \mu \mathrm{m} .{ }^{115,116}$ Atomic force microscopy (AFM) is a powerful technique that provides sub-atomic resolution of the surface morphology. ${ }^{117}$ This technique has also been used in combination with reflection interference contrast microscopy (RICM) which relies on interference patterns of reflected monochromatic light from a surface at different positions. ${ }^{118}$ Surface roughness has also been characterized by white-light interferometry with a resolution of $0.1 \mathrm{~nm} .{ }^{119,120}$ Scanning near-field optical microscopy (SNOM) is another surface characterization technique with a resolution in the nanometer size-range that could prove useful for micro- and nanoparticle characterization. ${ }^{121}$

Information about the formation, structure, and shape of MCs can be obtained in situ with high resolution by using small-angle X-ray scattering (SAXS), ${ }^{122} \mathrm{X}$-ray computed tomography (CT) ${ }^{123}$ or positron emission annihilation lifetime spectroscopy (PALS) for porous structures. ${ }^{124,125}$ For instance, Zetterlund et al. used SAXS to monitor in situ the multi-wall formation of methacrylate nanocapsules. Wide-angle X-ray diffraction (XRD) is a method of choice for the characterization of crystalline domains.

The composition of MCs can be measured by Fourier-transform infrared spectroscopy (FTIR), Raman spectroscopy, ${ }^{59,126}$ and thermogravimetric analysis (TGA). ${ }^{60}$ The mass of the encapsulated core is often measured after crushing the capsules in a mortar and extracting it in a solvent (e.g., soxhlet extraction). ${ }^{30,127}$ The mass ratio of the encapsulated core to the initially added core material corresponds to the encapsulation efficiency of the synthetic procedure. ${ }^{128}$ Elemental composition can also be extracted from X-ray photoelectron spectroscopy (XPS) ${ }^{129}$ and energydispersive X-ray spectroscopy (EDS). ${ }^{130}$ Time-of-flight secondary-ion mass spectrometry (TOF- 
SIMS) has been employed to characterize MC wall material by sputtering the surface with an ion beam. $^{131}$

The surface charge of MCs governs the stability of their suspensions, and can be obtained by measuring the zeta-potential (e.g., streaming potential, electrophoresis). ${ }^{132}$ Shi et al. measured the zeta potential of poly(urea-formaldehyde) (PUF) at $\mathrm{pH}$ values ranging from 1 to $12 .{ }^{133}$ The zeta potential decreased with increasing $\mathrm{pH}$ to about $-16 \mathrm{mV}$, becoming negative past the isoelectric point at $\mathrm{pH} 2.5$. Adsorption of polyelectrolytes at the surface of the MCs converted the zeta potential from negative to positive values.

The thermal properties of MCs play an important role in many applications, e.g., in phasechange or self-healing materials, and are typically measured by TGA and differential scanning calorimetry (DSC). ${ }^{134,135}$ From TGA measurements, Zhang et al. reported thermal stability for poly(melamine-formaldehyde) (PMF) MCs filled with $n$-octadecane up to ca. $160{ }^{\circ} \mathrm{C}$. The thermal stability was improved up to $230^{\circ} \mathrm{C}$ by promoting expansion space inside the MCs using a volatile component during the synthesis. ${ }^{136}$ The decomposition of PUF wall materials takes place around $240{ }^{\circ} \mathrm{C},{ }^{30,57,137}$ with a characteristic mass loss occuring at $100^{\circ} \mathrm{C}$ corresponding to the elimination of residual water and formaldehyde. This temperature limit is also noted for PUF microcapsules; however, a step-wise decomposition profile is generally reported. Above the decompsition temperature of cross-linked PUF, crack formation in the MC shell occurs. In addition, during the decomposition of the wall material, reaction with the core can take place and decrease the rate of decomposition of the ensemble. The encapsulation process provides protection to the core materials and therefore improves thermal stability. ${ }^{137}$

The mechanical properties of MCs are an essential parameter for many applications, and both bulk and individual capsule measurements have been performed, as summarized in Table 
3. ${ }^{138}$ Micromanipulation compression measurements were used to monitor the stress-strain relationship during the deformation of PMF microcapsules. ${ }^{139}$ The behavior of the material was proposed to correlate with an elastic-perfectly plastic model with strain hardening. The reported average failure strain was ca. 0.48 , and the failure stress ca. $350 \mathrm{MPa}$.

A major application of MCs is for the controlled and targeted release of active agents. Characterization of the barrier properties but also of the release profile is therefore of importance. The delivery of the core material can take place according to a burst-release mechanism by breakage of the capsule or dissolution of the shell, or according to a controlled-release mechanism by diffusion through the wall. ${ }^{21}$ Controlled release can furthermore take place continuously (extended-release) or intermittently (pulsatile release) over a period of time. The barrier properties of the wall are highly dependent on the wall thickness, and porosity. The total specific surface area can be measured with the Brunauer-Emmett-Teller (BET) analysis. This technique relies on the adsorption of nitrogen at varying pressures, and correlates the volume of adsorbed gas to the surface area of the microparticles. Pore volume and pore area distribution are obtained with the Barrett-Joyner-Halenda (BJH) analysis relying on nitrogen adsorption and desorption. ${ }^{140}$

The release profile of MCs is generally determined by dispersing of the particles in a solution and by measuring the evolution of the solute concentration over time. The release kinetics can be determined by a range of techniques adapted to the core content, such as spectroscopic techniques (UV-vis, ${ }^{141}$ NMR, FTIR spectroscopic methods), microscopy (fluorescence), ${ }^{142,143}$ chromatography $\left(\mathrm{GC},{ }^{114,144} \mathrm{HPLC}^{145}\right)$, sensors (e-nose ${ }^{146}$ ) and TGA. Indirect measurements can also be performed by measuring the effect of the released core materials (e.g., cytotoxicity) ${ }^{147}$.

Mercadé-Prieto et al. derived a theoretical method to calculate the permeability of MF microcapsules containing a hydrophobic liquid core. The release profile of single MCs was shown 
to be linear; however, the size polydispersity of the sample yielded an exponential-like decay at higher relative release ratios. The permeability in aqueous solutions with low molar mass alcohols ranged from 0.5 to $1 \times 10^{-12} \mathrm{~m}^{2} \mathrm{~s}^{-1}$.

\subsection{Amino resin composition}

The ratio of formaldehyde to urea and/or melamine has important effects on the resin properties, and the kinetics of polymerization. PUF microcapsules are generally prepared using a 1:1.9 molar ratio of urea:formaldehyde. ${ }^{58,112}$ Using a composition slightly lower than the stoichiometric ratio between the $-\mathrm{NH}_{2}$ groups and formaldehyde favors the complete functionalization of the formaldehyde. This composition was found to provide maximum encapsulation efficiency for UF microcapsules filled with pesticides. ${ }^{126}$ A molar ratio lower than 1:1.8 was noted to promote polymer precipitation upon acid addition. ${ }^{58}$

PMF microcapsules typically display greater mechanical strength and chemical resistance than PUF capsules. ${ }^{43}$ The multi-functionality of melamine favors network formation, and introduces hydrophobic and rigid aromatic rings into the structure. For the preparation of MF microcapsules, Kage et al. determined an optimal molar ratio of 1:3 for melamine:formaldehyde, which corresponds to the stoichiometric ratio between the $-\mathrm{NH}_{2}$ groups and formaldehyde. ${ }^{148}$ Improved properties can also be obtained by the addition of melamine to UF (typically $6-8 \mathrm{wt}$ $\%) .{ }^{55}$ In some instances, MUF capsules are prepared by mixing separate batches of precondensate composed of UF and MF respectively. ${ }^{43}$ The order of addition in this case is of importance as the MF precondensate was found to destabilize the dispersion. 


\subsection{Core material}

The encapsulation process relies on a low degree of interactions and efficient phase separation between the core and shell materials. Hydrophobic compounds are most suitable for encapsulation by amino resins, and have been used in a wide range of applications. Reactive compounds (e.g., pharmaceuticals, adhesives, cosmetics) can also be encapsulated by prior dissolution, dispersion or suspension in a hydrophobic medium.

A wide range of compounds has been encapsulated for applications including pressuresensitive recording materials, ${ }^{149}$ adhesives,${ }^{52}$ agrochemicals, ${ }^{147}$ perfumes, ${ }^{150}$ vegetables oils, ${ }^{151}$ consumer products, flame retardants, ${ }^{152}$ phase change materials, ${ }^{153}$ electronic inks, ${ }^{154}$ thermosensitive paper ${ }^{155}$ self-healing agents, ${ }^{53}$ drag-reducing agents, ${ }^{135}$ smart coatings,${ }^{54}$ and polymer additives. ${ }^{33}$ Core materials such as epoxy resins, for instance, are high performance adhesives and promising candidates for self-healing applications. ${ }^{56,59,60,156}$ These reactive compounds were found suitable for the in situ encapsulation process, and did not react with the amino resin even at a low $\mathrm{pH}^{30,59}$ Other compounds such as fragrance oils, ${ }^{150}$ corrosion inhibitors, ${ }^{157}$ electrophoretic fluids, ${ }^{158}$ chlorophyll, ${ }^{159}$ and glass beads ${ }^{160}$ have also been successfully encapsulated with amino resins.

In practice, the suitability of materials towards encapsulation is often determined empirically. However, some theoretical models have been suggested to guide the selection process. Sliwka proposed to consider Hildebrand's solubility parameters $(\delta),{ }^{161}$ which provide a semiquantitative assessment of the component interactions. ${ }^{12}$ Solubility parameters as different as possible are desirable for the preparation of impermeable MCs. Hansen extended Hildebrand's expression by introducing separate parameters for dispersive $\left(\delta_{d}\right)$, dipolar $\left(\delta_{p}\right)$ and hydrogenbonding $\left(\delta_{h}\right)$ interactions, such that $\delta=\delta_{d}+\delta_{p}+\delta_{h \cdot}{ }^{162}$ Based on Hansen solubility parameters 
(HSP), Stöver et al. recommended a value for the core material 3-8 $\mathrm{MPa}^{1 / 2}$ below that of the polymer for the encapsulation of alcohols in polyurea MCs by interfacial polymerization. ${ }^{163}$ Hofmeister et al. demonstrated a linear relationship between $\delta_{h}$ and the encapsulation efficiency in nanocapsules prepared with an acid-functionalized acrylate copolymer. This work allowed the prediction of the encapsulation efficiency for various volatile compounds and their mixtures. The encapsulation of otherwise poorly encapsulated materials was improved by adding a mediator compound with a low $\delta_{h}$ value. ${ }^{164}$ Latnikova et al. demonstrated that HSP can be used to predict the morphology of MCs prepared by interfacial polymerization. The affinity between the polymer and the core material directed the formation of a core-shell, a multi-compartment, or a compact morphology. ${ }^{165}$

Table 4 compares the solubility parameters of UF resin $\left(\delta=25.7 \mathrm{MPa}^{1 / 2}\right)$ to selected compounds. From the HSP of a pair of compounds 1 and 2, one can determine the solubility parameter distance $R_{\mathrm{a}}$, as given by Equation 2:

$$
R_{a}^{2}=4\left(\delta_{d 1}-\delta_{d 2}\right)^{2}+\left(\delta_{p 1}-\delta_{p 2}\right)^{2}+\left(\delta_{h 1}-\delta_{h 2}\right)^{2}
$$

The ratio of $R_{\mathrm{a}}$ to the radius of the solubility sphere $R_{0}$ (obtained experimentally) yields the relative energy difference term RED, which provides information on the affinity between two compounds. A $R E D$ number lower than 1 denotes high affinity, while values progressively greater than unity signify lower affinities. The $R_{0}$ values for selected materials, and the corresponding $R_{\mathrm{a}}$ and $R E D$ values with respect to UF resins are indicated in Table 4.

\subsection{Wall formation}

\subsubsection{Mechanism of wall formation}

The mechanism of capsule wall formation was investigated by Brown et al. in the encapsulation of dicyclopentadiene with PUF. ${ }^{112}$ By monitoring the MC formation by optical 
microscopy and the evolution of the $\mathrm{pH}$ and temperature during the reaction, the authors uncovered four distinct phases (Figure 8a). The process started with the dispersion of the core materials in the aqueous solution in presence of the polymer precursor. The polymerization reaction was initiated by increasing the temperature and lowering the $\mathrm{pH}$ of the solution. During the second phase, soluble oligomeric species formed and deposited at the core/water interface as a smooth layer, while the emulsion solution turned cloudy. The interfacial tension between the hydrophobic core and aqueous continuous phase acted as the driving force for the deposition of polymer as a smooth layer until reaching a limit. ${ }^{160,166}$ The third stage was marked by further polymer deposition, and the nanoprecipitation of the amino compounds both at the surface of the MCs and in solution. Growth of the capsule wall then continued by formation of a porous and rough surface. Scattering from the large aggregates turned the solution milky-white. This process was driven by the decreased solubility of the polymeric and colloidal species present in solution (Section 2.4). In the last phase of the reaction, the completion of the curing reaction was indicated by the clarification of the solution. The MCs were then easily separable from the reaction medium. This phenomenological description accounts for the wall formation, however, details about the underlying mechanism of microcapsules formation remains subject to discussion. In Section 4, proposed mechanisms are further discussed invoking a strictly in situ mechanism or elements of complex coacervation and interfacial polymerization.

\subsubsection{Wall thickness}

The wall formation during the different regimes typically yields a binary surface composed of a smooth inner membrane, and a porous outer surface. ${ }^{11,112}$ The wall thickness of the inner membrane was found to be independent of the processing parameters, and ranged from 150 to 300 
$\mathrm{nm}$ (Figure 8b) ${ }^{60,111,112,160}$ The outer layer thickness however depended on the nanoparticle (NP) formation process, and was affected by the rate of UF condensation reaction (i.e., $\mathrm{pH}$, temperature, concentration), the ratio of core to wall material, and the mixing dynamics. ${ }^{160}$ The porous outer layer increased the mechanical strength and the adhesion of the capsules but could display adverse effects on the optical properties in some applications. ${ }^{167-169}$

A comparable mechanism was noted during the preparation of MF microcapsules. ${ }^{148,170}$ Sgraja et al. determined the capsule shell thickness from density measurements, and monitored its evolution as a function of wall material concentration per volume of dispersed phase $\left(m_{\mathrm{w}} / V_{\mathrm{d}}\right) .{ }^{171}$ For a core (tetradecene) volume fraction of 0.29 , a plateau was observed above $100 \mathrm{~g} \cdot \mathrm{L}^{-1}$, which indicated the maximum wall concentration required for the encapsulation. Smooth capsules were obtained at concentrations below this limit. When using an excess of wall materials, NPs formed and deposited at the surface of the capsules. By taking into account the specific surface area of the emulsion, the shell thickness was plotted against the wall concentration over the total surface area of the droplets (Figure 9). The shell thickness was found to increase linearly with the wall concentration up to a critical size of $100 \mathrm{~nm}$, which corresponded to the limit of smooth surface formation. In contrast to UF and MF microcapsules, MUF capsules were more resilient to the formation of NPs, and displayed a smooth and relatively thicker wall up to $700-900 \mathrm{~nm}^{32}$

\subsubsection{Nanoporous surface}

As indicated above, the formation of NPs in solution is dependent on the rate of polycondensation. An increase in the rate of polymer formation shortens the second stage of the encapsulation process (deposition on the core surface), and results in a greater formation of nanoprecipitate. Control over the kinetics of the reaction can be obtained by varying the 
temperature or the $\mathrm{pH}$ of the solution. For instance, an increase in the reaction temperature from $50{ }^{\circ} \mathrm{C}$ to $70{ }^{\circ} \mathrm{C}$ during the encapsulation of palm oil by UF was reported to increase the NP formation, and to reduce the encapsulation efficiency by over $80 \% .{ }^{172}$ Chuanjie et al. studied the effect of the heating rate during the one-step encapsulation of tetrachloroethylene by UF, and noted the formation of a rough capsule surface when the heating rate was above $0.5^{\circ} \mathrm{C} / \mathrm{min}$ (conditions: $\mathrm{pH}\left(\right.$ initial) 3.5 , [UF resin] $\left.=36 \mathrm{~g} \cdot \mathrm{L}^{-1},[\mathrm{NaCl}]=50 \mathrm{~g} \cdot \mathrm{L}^{-1}\right) .{ }^{111}$ A marked decrease in the yield of the encapsulation was also observed with a heating rate above $1{ }^{\circ} \mathrm{C} / \mathrm{min}$. On the other hand, in the preparation of epoxy-encapsulated UF (epoxy@UF) MCs at $60{ }^{\circ} \mathrm{C}$ and $40{ }^{\circ} \mathrm{C}$, Cosco et al. revealed that the low reaction temperature adversely affected the efficiency. ${ }^{59}$ It was presumed that a poorer dispersion of the core material resulted in polymer precipitation in solution rather than at the core surface.

The $\mathrm{pH}$ also affects the kinetics of polymer formation. In a one-step process, two competing reactions take place at the beginning of the reaction: 1) The formation of water-soluble and surface active methylol ureas, and 2) the polycondensation reaction yielding an insoluble polymer. Fan et al. encapsulated glass beads with a UF wall in the presence of $\mathrm{NH}_{4} \mathrm{Cl}$, and varied the initial $\mathrm{pH}$ from 2.5 to $4.5 .{ }^{160}$ Smooth MCs were obtained when the initial $\mathrm{pH}$ value was 4.5. Upon lowering of the $\mathrm{pH}$, NPs formed but remained in suspension and did not deposit on the surface of the capsules. MCs with a rough surface were obtained when the initial $\mathrm{pH}$ value was 3.5 or lower. A relatively high final $\mathrm{pH}$ (above $\mathrm{pH}$ 3) was also conducive to smooth capsule wall formation. In another study, Brown et al. showed that by maintaining a constant $\mathrm{pH}$ value $\mathrm{pH}$ 3.5), smooth capsules were obtained, and the UF NPs remained in suspension. ${ }^{112}$ 


\subsection{Core/wall ratio and viscosity}

The amount of core material affects the dispersion process and determines the surface area available to the wall material under specified conditions. The initial core/wall ratio is therefore of importance during the encapsulation process. In the preparation of epoxy@UF MCs, the size was reported to increase from $203 \mu \mathrm{m}$ to $238 \mu \mathrm{m}$ when the core/wall ratio increased from 1.2 to 1.4 , and a broader size distribution was noted. ${ }^{56}$ A higher core content promoted the formation of larger droplets in solution.

An increase in core material was found also to result in a thinner and smoother capsule wall, which exhibited weaker mechanical strength and resulted in MC fracture during material handling. ${ }^{56}$ It is expected that the dispersion of a larger amount of core material would be more difficult, as droplet coalescence takes place more readily. As reported by Liang et al, the yield decreased from $80 \%$ to $70 \%$ during the encapsulation of epoxy by UF when the core/wall ratio increased from 0.5 to $2.0 .^{30}$ The optimal ratio found in the study was 1.25 (other conditions: U/F: $0.5 \mathrm{w} / \mathrm{w}, 380 \mathrm{rpm}$, surfactant, $\mathrm{pH} 3-4,60-65{ }^{\circ} \mathrm{C}$, reaction time $\left.3 \mathrm{~h}\right) .{ }^{30}$

A correlation between the viscosity of the core material and its dispersion can also be expected. In the preparation of UF and MF microcapsules for instance, the size was found to increase when the viscosities of the core materials (fragrance oils) increased. ${ }^{150,173}$ It was argued that a higher surface tension resulted in larger droplet size during the emulsion. When the viscosity was greater than $30 \mathrm{cP}$ however, droplet coalescence was instead hindered, resulting in smaller capsule sizes. In a water-in-oil emulsion, Sanghvi and Nairn investigated the influence of the viscosity ratio between the dispersed phase and the continuous oil phase. ${ }^{174}$ The increase in MC size was also found to occur, but only above a critical viscosity ratio. At high viscosity ratio, the 
break-up of the droplet was deemed more difficult and yielded larger MCs. Unstable dispersions also resulted when the viscosity of the dispersed phase was too high.

\subsection{Orthogonal experiments}

The microencapsulation process relies on synergistic contributions of multiple factors, and depends on the composition of the formulation. ${ }^{33}$ The many variables involved in the encapsulation process make it difficult to assign a particular outcome to the individual parameters. In order to address this issue, several groups used orthographic factorial design to determine the optimal experimental conditions for the preparation of capsules. ${ }^{56,111,175}$

For the encapsulation of two-phase core materials, Zhao et al. selected four variables and used the viscosity of the solution as a determining parameter. ${ }^{175}$ The order of influence of the variables, and the optimum conditions established are shown in Table 5. The choice of the determining parameter was important, and affected the ranking of the variables. By studying the formation of epoxy@UF microcapsules, Wang et al. selected four different determining parameters: yield, core content, capsule diameter, and shell thickness. ${ }^{56}$ The resulting ranking of the variables and their optimum values are indicated in Table 5. Chuanjie et al. determined the encapsulation yield for tetrachloroethylene@UF by separating the MCs from the undeposited polymeric materials. ${ }^{111}$ The separation of the products was based on the difference in settling velocity owing to the density difference between the MCs and the precipitated polymer $\left(1.60 \mathrm{~g} \cdot \mathrm{mL}^{-}\right.$ ${ }^{1}$ and $1.05 \mathrm{~g} \cdot \mathrm{mL}^{-1}$, respectively). Using this parameter, optimum conditions were also established (Table 5). The diversity of optimum conditions reported highlights the need to adjust each variable according to a particular encapsulation process. The selection of the parameters also depends on the choice of the specific determining parameter of interest. 


\subsection{Control of surface properties}

\subsubsection{Two-step and one-step protocols}

The formation of PUF microcapsules relies on the efficient phase separation between the core and wall materials during encapsulation. As discussed above, the difference between the solubility parameters of the materials dictates the partition process. The synthetic scheme generally follows a two-step protocol starting with the preparation of a UF precondensate under alkaline conditions. The prepolymer may display surface active properties promoting the dispersion of a core material and its encapsulation under acidic conditions. The addition of surfactants has been found to provide additional control over the interfacial interactions, and to limit Ostwald ripening and droplet coalescence. ${ }^{176}$ The surfactant can serve various roles, including as 1) accelerator for the dispersion of the core phase, 2) stabilizer for the emulsion, 3) accelerator for the wallformation, and 4) stabilizer for the MCs. ${ }^{35}$ More stable dispersions allow the preparation of MCs in a one-step encapsulation protocol. In this case, the preparation of a precondensate solution prior to the encapsulation process is not required. ${ }^{12,132,167}$

\subsubsection{Surface tension}

The interfacial tension is related to the work required to form new surfaces, and will influence the surface area and stability of the emulsion droplets. The equilibrium of a dispersion of two immiscible liquids (phase-1 and phase-3) in a third immiscible liquid (phase-2) can be expressed as a function of the interfacial tensions $\gamma_{\mathrm{ij}}$ and spreading coefficients $\left(S_{\mathrm{i}}=\gamma_{\mathrm{ij}}-\left[\gamma_{\mathrm{ij}}+\gamma_{\mathrm{ij}}\right]\right)$. Torza and Mason reported three sets of relations to account for the configuration of the system when $\gamma_{12}>\gamma_{23}\left(\mathrm{~S}_{1}<0\right)$, as shown in Figure $10 .{ }^{177}$ Complete phase encapsulation of phase-1 in 
phase-3 is established when $\mathrm{S}_{3}<0$, while phase separation occurs when $\mathrm{S}_{2}>0$. Only partial encapsulation is observed when all the spreading coefficients are negative.

In a model proposed by Van Oss, ${ }^{178}$ the solubility $(S)$ of a polymer (2) in a solvent (1) is related to the free energy of interfacial interaction $\left(\Delta G_{212}\right)$, and is given by Equation $3 .{ }^{179}$ The latter parameter is a function of the interfacial interaction parameters for each component, and takes into account Lifshitz-van der Waals and Lewis acid-base forces. Similar expressions are derived for a ternary system involving two distinct materials (2 and 3) dispersed in a solvent (1).

$$
R T \ln (S)=f\left(\Delta G_{212}\right)
$$

\subsubsection{Amino resin surface activity}

The surface activity of UF resins appears somewhat limited. Dietrich et al. reported no significant interfacial tension between UF resins in water against xylene. ${ }^{166}$ On the other hand, Guo et al. determined modest surfactant properties for UF prepolymers by measuring a decrease in interfacial tension (from $43 \mathrm{mN} \cdot \mathrm{m}^{-1}$ to $35 \mathrm{mN} \cdot \mathrm{m}^{-1}$ ) between water and tetrachloroethylene. ${ }^{180}$ The authors argued that the surface activity of the oligomeric species promoted the encapsulation process. Additives such as ammonium chloride can also help to increase the surfactant properties of the UF polymers by promoting the formation of amine groups (Section 3.9.2). ${ }^{132} \mathrm{MF}$ resins have been demonstrated to exhibit more pronounced surfactant properties. ${ }^{166,171}$ For instance, Sgraja et al. measured the surface tension of MF resins which decreased below $45 \mathrm{mN} \cdot \mathrm{m}^{-1}$ at a concentration above $50 \mathrm{~g} \cdot \mathrm{L}^{-1}$, and an interfacial tension between the resin and tetradecene below $12.2 \mathrm{mN} \cdot \mathrm{m}^{-1}$ at a concentration above $10 \mathrm{~g} \cdot \mathrm{L}^{-1}$ (Figure 11$)$.

Resins etherified by reaction with methanol displayed an enhanced surface activity. ${ }^{166}$ Even better surfactant properties were obtained in the presence of an amino alcohol modifier such 
as triethanolamine (Figure 12). Salaün et al. monitored the surface tension during the encapsulation of $n$-hexadecane with etherified MF, and noted three different stages (Figure 13). ${ }^{181}$ Upon acid addition at the beginning of the reaction, protonation of the prepolymer increased the degree of etherification of the polymer. The ensuing drop in solubility lowered the surface tension of the solution. In the second stage, deposition of the polymer at the interface of the dispersed phase resulted in the recovery of the surface tension of the solution. The last stage was marked by a further decrease in the surface tension upon continuous polycondensation reactions taking place primarily at the surface of the MCs.

\subsubsection{Hydrophilic-lipophilic balance}

The ability of a surfactant to promote the emulsion of a core phase can be established on the basis of the balance of its hydrophilic and hydrophobic character. Griffin introduced a classification method for surfactants based on these parameters, coined the hydrophile-lipophile balance (HLB) method. ${ }^{182,183}$ The HLB value is related to the weight percentage of the hydrophilic segment of a surfactant and can be calculated or determined experimentally. ${ }^{184-186}$ For instance, as shown in Equation 4, Griffin proposed to calculate the HLB value of non-ionic surfactants by taking into account the molar masses of their hydrophilic $\left(M_{\mathrm{H}}\right)$ and lipophilic $\left(M_{\mathrm{L}}\right)$ segments. ${ }^{183}$ This equation can equally be expressed as a function of the molar volume $V_{\mathrm{H}}\left(V_{\mathrm{L}}\right)$ and density $\rho_{\mathrm{H}}$ $\left(\rho_{\mathrm{L}}\right)$ of the hydrophile (lipophile) moieties, respectively. A low HLB value $(<9)$ is indicative of an affinity for non-polar solvents, while high values $(>11)$ characterize hydrophilic species. Stable emulsions can be obtained by matching the HLB value of the surfactant to that of the dispersed phase (required HLB). As illustrated in Table 6, the characteristics required for different applications correspond to a range of HLB values. 


$$
H L B=20 \times \frac{M_{H}}{M_{H}+M_{L}}=20 \times \frac{V_{H} \rho_{H}}{V_{H} \rho_{H}+V_{L} \rho_{L}}
$$

For the encapsulation of epoxy with UF polymers, Wang et al. recommended a HLB value for the surfactant ranging from $8-18$, which is characteristic of oil-in-water $(\mathrm{O} / \mathrm{W})$ emulsion systems. ${ }^{56}$ The encapsulation of water-soluble core materials, on the other hand, can be performed from water-in-oil (W/O) emulsions. Using UF polymers, Golden recommended a HLB value for the surfactant in the range of $2-8 .^{187}$ The encapsulation of chlorophyll with UF polymer was obtained from multiple emulsions (W/O/W) and involved sorbitan oleate (Span 80) and polyoxyethylene sorbitan monooleate (Tween 80) with HLB values of 4.3 and 15.0, respectively. ${ }^{159}$ The emulsification of $n$-hexadecanol using surfactants with HLB values near 15 was deemed ineffective, however, and larger molar mass stabilizers were preferred. ${ }^{188}$

It is noteworthy that a correlation has been drawn between the solubility parameters (Section 3.4), and the HLB value. This was applied to predict the formation of microparticles. ${ }^{189}$ For instance, Little proposed the empirical relationship shown in Equation 5 for a series of surfactants between the HLB values and Hildebrand parameter $\delta\left(\right.$ in $\left.\mathrm{MPa}^{1 / 2}\right) \cdot{ }^{190}$

$$
H L B=54 \times\left(\frac{\delta-16.8}{\delta-12.3}\right)
$$

Beerbower et al. rather determined the HLB values from Hansen's solubility parameters for dispersive $\left(\delta_{d}\right)$, dipolar $\left(\delta_{p}\right)$ and hydrogen-bonding $\left(\delta_{h}\right)$ interactions. ${ }^{191}$ Using Griffin's definition (Equation 4), the authors expressed the cohesive energy ratio $R_{\mathrm{c}}$, as proposed by Winsor (Equation 6), ${ }^{192}$ as a function of the HLB value (Equation 7).

$$
R_{c}=\frac{V_{L}\left(\delta_{d}^{2}+0.25 \delta_{p}^{2}+0.25 \delta_{h}^{2}\right)_{L}}{V_{H}\left(\delta_{d}^{2}+0.25 \delta_{p}^{2}+0.25 \delta_{h}^{2}\right)_{H}}
$$




$$
R_{c}=\frac{\rho_{H}}{\rho_{L}} \times\left(\frac{20}{H L B}-1\right) \times \frac{\left(\delta_{d}^{2}+0.25 \delta_{p}^{2}+0.25 \delta_{h}^{2}\right)_{L}}{\left(\delta_{d}^{2}+0.25 \delta_{p}^{2}+0.25 \delta_{h}^{2}\right)_{H}}
$$

The surfactant is selected with the aim to promote chemical compatibility between the hydrophilic moiety and the water phase, and between the lipophilic moiety and the oil phase. The required $H L B_{0}$ for the oil phase can be estimated from Equation 8:

$$
H L B_{0}=\frac{20}{1+R_{c} \frac{\rho_{L}}{\rho_{H}} \frac{\left(\delta_{d}^{2}+0.25 \delta_{p}^{2}+0.25 \delta_{h}^{2}\right)_{H}}{\left(\delta_{d}^{2}+0.25 \delta_{p}^{2}+0.25 \delta_{h}^{2}\right)_{L}}}
$$

\subsubsection{Surfactant and microcapsule synthesis}

A variety of surfactants have been used for the preparation of amino resin MCs, including sodium dodecyl sulfate (SDS), sodium dodecylbenzene sulfonate (SDBS), and octylphenol ethoxylates (OP). Natural polymers have similarly been used, and encompass gum arabic, ${ }^{193}$ alginic acid, cellulose, ${ }^{168}$ gelatin, starch, lignin, and their derivatives (e.g., carboxymethylated, sulfated, phthalated, sulfonated). Synthetic polymers have found widespread use and include copolymers of maleic anhydride, ${ }^{194-196}$ polyacids such as polymers containing (meth)acrylic acid, and vinylbenzenesulfonic acid, ${ }^{193,197}$ polyesters and polyacrylamides, ${ }^{43}$ (carboxyl-functionalized) polyvinyl alcohols, and polyiso(thio)cyanates. ${ }^{35,198}$ Poly(ethylene glycol) acts as a stabilizer according to a depletion-stabilization mechanism. ${ }^{171}$ Cross-linkable surfactants can also be obtained from the amino resin itself by etherification with alcohols. ${ }^{199}$

Under certain circumstances, the addition of surfactants can alternately promote or hinder the encapsulation process. In the preparation of epoxy-filled PUF microcapsules, Yuan et al. investigated the influence of surfactants including SDBS, OP, SDS, and styrene-maleic anhydride copolymer (SMA). Only SDBS was found to improve the encapsulation process, and promote the 
formation of capsules with smooth surfaces. The authors argued that an increase in the solution viscosity after addition of OP or SMA hindered the polymer deposition. Smaller capsules with a narrower size distribution were obtained with increasing SDBS concentration, although an enhanced surface roughness was noted. An increase in viscosity stemming from a rise in electrostatic repulsions between the surfactant-stabilized species was invoked to account for the particle roughness.

Low molar-mass surfactants, such as SDS and SDBS, have a relatively low surface coverage, and show inconsistent effects. ${ }^{150,168}$ However, such compounds find applications as costabilizers when used in combination with other surfactants. For instance, complexes of SDBS with gum arabic, ${ }^{167}$ or SDS with sodium carboxymethyl cellulose (CMC $)^{168}$ showed improved performance as stabilizers when compared to their individual components.

The ratio between the surfactant and co-surfactants was found critical to promote phase separation and wall formation. Salaün et al. reported better performance when using a mixture of Tween-20 (polyoxyethylene sorbitan monolaurate), and Brij-35 (polyoxyethylene lauryl ether) surfactants rather than either individual surfactant. ${ }^{181}$ Attractive interactions between the polar heads of the surfactants were thought to yield synergistic interactions at the interface. As shown in Figure 14, the interaction of the surfactant with the acid affected the surface tension of the solution, and displayed a minimum for a $\mathrm{pH}$ value dependent on the $\mathrm{pKa}$ values of the acid ([pKa $\left.\left.+\mathrm{pKa}_{2}\right] / 2\right)$.

Yoshizawa et al. reported that surfactants such as poly(ethylene-alt-maleic anhydride) (poly(E-MA)) in addition to stabilizing the oil phase dispersion, introduced reactive sites that promoted the condensation reaction of urea and formaldehyde. ${ }^{200}$ Inert surfactants such as SDS and poly(vinyl alcohol) were found ineffective for the $\mathrm{MC}$ formation, while oil-soluble compounds 
(Solsperse 17,000) hindered the deposition of other surfactants and prevented MC formation (Figure 15). ${ }^{201}$ Reactive functional groups such as maleic anhydride were deemed preferable over carboxylic acid groups in comparable copolymers. Notably, the minimum concentration of poly(EMA) required for encapsulation corresponded to the critical micelle concentration of the surfactant. ${ }^{200}$ The deposition of the surfactant at the core/water interface was promoted for systems displaying large interfacial activity and high molar masses.

Hoshi and Matsukawa also reported that the introduction of reactive sites in the oil phase, by using polyvalent iso(thio)cyanates, could improve the colloidal stability and capsule formation. ${ }^{198}$ During the preparation of MF microcapsules, Powell employed a styrene-maleic acid copolymer and suggested that an interfacial reaction mechanism took place between the amino resin and the copolymer, favoring $\mathrm{MC}$ formation.

Amphiphilic compounds such as poly(E-MA) were found to deposit at the surface of the core materials and catalyze polymer precipitation around the polymer. A hedgehog morphology resulted during the encapsulation of glass beads (Figure 16). ${ }^{160,167}$ By increasing the concentration of the amphiphile, the colloidal stabilization of UF NPs occurred instead and promoted the formation of smooth MCs.

\subsection{Modified amino resins}

\subsubsection{Nanoadditives}

In addition to surfactants, other additives may be employed to promote the formation of capsules and to improve their properties. Modifiers such as nanomaterials, salts, and cross-linkers have been reported. As a general rule, the amount of modifier ranges from $0.75 \mathrm{wt} \%$ to $10 \mathrm{wt} \%$. 
Nanomaterials can be incorporated in the capsule wall during synthesis, and yield hybrid capsules with unique properties. For example, aluminum oxide NPs, ${ }^{202}$ silver NPs, ${ }^{203}$ titanium oxide NPs, ${ }^{204}$ or single-walled carbon nanotubes ${ }^{202}$ have been used to increase the mechanical strength, ${ }^{205}$ and the thermal and water resistance of PUF microcapsules. The presence of the nanomaterials was found to decrease the surface roughness by controlling the rate of the polymerization reaction, and to produce smaller capsules. ${ }^{205}$ Sun et al. proposed a model for PUF microcapsules formation, and highlighted the role of hydrophobic NPs in the stabilization of the dispersion by deposition at the core/wall interface (Figure 17). The incorporation of iron NPs yielded MCs with magnetic properties that could be collected using a magnet (Figure 18a, b). ${ }^{206}$ The in situ polymerization of urea and formaldehyde was also favorable for the exfoliation of nanoclays, that once incorporated, increased the barrier properties of the capsules by increasing the diffusion path ( $p$ and $p^{\prime}$ ) of a core material (Figure 18c). ${ }^{169}$

\subsubsection{Salts}

Stronger capsule walls and improved barrier properties were obtained by addition (2-20 wt $\%$ ) of the water soluble salt of a strong acid and a strong base (typically $\mathrm{NaCl}$ ) ${ }^{49}$ When added to tetrachloroethylene@UF MCs, $\mathrm{NaCl}$ improved the core retention by $30 \%$ at a concentration of 25 $\mathrm{g} \cdot \mathrm{L}^{-1}$, but only limited increased benefit was noted above this concentration. ${ }^{111}$ Similarly, lower viscosity and improved core retention were noted upon addition of $1-10 \mathrm{wt} \%$ of salts composed of cations from the group 1 elements, and chloride, sulfate, phosphate, and nitrate anions (particularly $\left.\mathrm{KH}_{2} \mathrm{PO}_{4}\right){ }^{207}$ The addition of salt increased the ionic strength of the solution, and was thought to promote aggregation and surface deposition by destabilizing the double electric layer of the colloidal UF species (Section 2.4). Additionally, in the presence of a negatively charged 
surfactant such as gum arabic, electrolytes (e.g., $\mathrm{NaCl}$ ) were hypothesized to interact with the charged compounds and to promote greater surface activity. ${ }^{111}$

Reactive compounds such as $\mathrm{NH}_{4} \mathrm{Cl}$ have been found to yield PUF microcapsules with low permeability and improved mechanical strength, heat resistance, and shelf life. ${ }^{160,197}$ In addition to disrupting the electric double layer, ammonium chloride may act as an acid catalyst during the reaction and lower the $\mathrm{pH}$ of the solution (Figure 19, Equations 9-10). The $\mathrm{pH}$ change provides control over the rate of polymerization (Scheme 5). Further investigation by Fan et al. suggested that the reaction between $\mathrm{NH}_{4} \mathrm{Cl}$ and the UF resin may also form surface active species that promote UF nanoparticle deposition at the surface of the dispersed phase (Equation 11). ${ }^{132,208}$

$$
\begin{aligned}
4 \mathrm{NH}_{4} \mathrm{Cl}+6 \mathrm{HCHO} & \rightleftharpoons\left(\mathrm{CH}_{2}\right)_{6} \mathrm{~N}_{4}+4 \mathrm{HCl}+6 \mathrm{H}_{2} \mathrm{O} \\
\mathrm{NH}_{4}^{+}+\mathrm{H}_{2} \mathrm{O} & \rightleftharpoons \mathrm{NH}_{3} \cdot \mathrm{H}_{2} \mathrm{O}+\mathrm{H}^{+}
\end{aligned}
$$

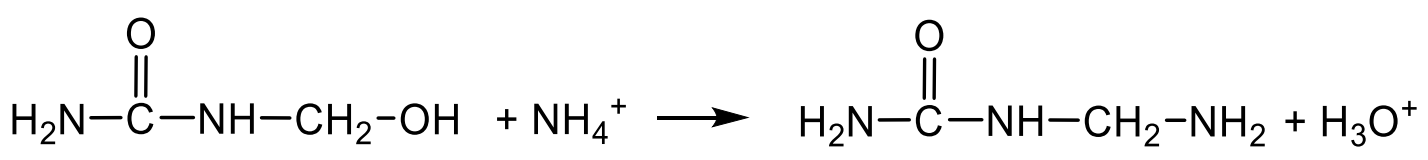

\subsubsection{Other additives: cross-linkers, hydrophobes, and scavengers}

The addition of a cross-linker during the capsule wall formation can improve the mechanical strength, and reduce the emission of free formaldehyde. Polyhydroxy compounds can react with urea and formaldehyde, and incorporate into the wall structure. Resorcinol is commonly used during the preparation of UF capsules. For instance, fast reaction of resorcinol with UF promoted network formation, and imparted epoxy@UF microcapsules with improved water resistance. ${ }^{59,209}$ Carboxymethyl cellulose displays a crystalline structure, and was used in the 
preparation of paraffin@UF MCs. ${ }^{210}$ Good mechanical resistance, and thermal stability were obtained.

Another method to increase the capsule water resistance is through the addition of hydrophobic additives such as waxes. ${ }^{28}$ Ultrahydrophobes (e.g., hexadecane, octane) have been added to the core material and used as co-stabilizers during the encapsulation process. ${ }^{211}$ The increased hydrophobicity reduced the Ostwald ripening process and yielded nanosized MCs upon sonication and stirring. Capsules as small as $220 \mathrm{~nm}$, with a shell thickness of $20 \mathrm{~nm}$ were thus obtained.

The emission of formaldehyde upon hydrolysis of the amino resin has long been regarded as a drawback in commercialization. Formaldehyde has been classified as carcinogenic to humans, ${ }^{212}$ and its use is strictly regulated. In order to limit the emission of formaldehyde from aminoplast capsules, scavengers have been added to the formulation. The most common scavengers such as urea, ammonia, melamine, and 2-cyanoguanidine are primary or secondary amines or their derivatives. ${ }^{35,213,214}$ More recently, sulfur-based compounds have proved to be effective scavengers. ${ }^{215}$ For instance $\mathrm{K}_{2} \mathrm{SO}_{3}$ reacts with formaldehyde to form a bisulfite adduct (Equation 12), and was able to prevent the discoloration of formulations containing MCs. ${ }^{216}$

$$
\mathrm{HCHO}+\mathrm{K}_{2} \mathrm{SO}_{3}+\mathrm{H}_{2} \mathrm{O} \rightleftharpoons \mathrm{KOH}+\mathrm{KSO}_{3} \mathrm{CH}_{2} \mathrm{OH}
$$

\subsection{Stirring rate and stirrer geometry}

Dispersion of the core material during the in situ polymerization relies on the adequate mixing of two immiscible phases and is typically achieved by mechanical stirring. A minimum shear rate is required for the formation of an emulsion. Above this minimum, the size of the droplets is inversely related to the stirring rate. A higher shear rate increases the turbulent kinetic 
energy provided to the system, and increases the rate of droplet dissociation. ${ }^{181}$ Several expressions have been suggested to account for the change in particle size with the stirring rate.

The size and size distribution of droplets can be predicted on the basis of the Weber number theory that determines the average equilibrium droplet size in a turbulent continuous phase. ${ }^{217}$ The droplet size is governed by the balance between disruptive forces (associated with the turbulent flow), and cohesive forces (associated with the interfacial tension and viscosity). From the proposition of local isotropy by Kolmogorov, ${ }^{218}$ a relationship can be established between droplet diameter (related to the Sauter diameter, $\left.d_{3,2}\right)^{219}$ and the droplet Weber number $\left(W_{e}\right)$. This is shown in Equations 13 and 14, where $C_{l}$ is a constant, $L$ is the stirrer diameter, $\rho_{j}$ the density of the continuous phase, $\omega$ the stirring rate, and $\gamma$ the interfacial tension.

$$
\begin{gathered}
\frac{d_{3,2}}{L}=C_{1} W_{e}^{-3 / 5} \\
W_{e}=\frac{\rho_{j} \omega^{2} L^{2}}{\gamma}
\end{gathered}
$$

By taking into account the decay of the turbulence, Equation 15 can be used to describe the change in droplet diameter with the stirring rate, where $\phi_{i}$ is the volume fraction of the core material, and $C_{2}$ a proportionality constant. The density $\rho$ can further be expressed as the average density of the system if premixed phases are employed (Equation 16).

$$
\begin{gathered}
d_{3,2}=C_{1}\left(\gamma^{-1} \omega^{2} L^{4 / 3} \rho\right)^{-3 / 5}\left(1+C_{2} \phi_{i}\right) \\
\rho=\rho_{j}\left(1-\phi_{i}\right)+\rho_{i} \phi_{i}
\end{gathered}
$$

This model was applied by Sgraja et al. ${ }^{171}$ to describe the in situ polymerization of PMF microcapsules. The authors demonstrated the dependence of the capsule size to the stirring speed, by slightly adjusting the value of the exponent to account for deviation from a perfectly local 
isotropic turbulence. From surface tension measurements (Section 3.8.3), it was found that above a resin concentration of $30 \mathrm{~g} \cdot \mathrm{L}^{-1}$, the surface activity was constant and thus yielded a constant droplet size distribution.

Dobetti and Pantaleo proposed to consider the hydrodynamic model of Armenante and $\operatorname{Kirwan}^{220}$ in the case of small particles $(<30 \mu \mathrm{m})$, to describe the formation of MCs from microeddies formed in the vicinity of the propeller during agitation. ${ }^{221}$ The size of the microeddies increases in regions far from the propeller, and also results in a droplet size and size-distribution inversely proportional to the stirring rate $(\omega)$. The minimum diameter of the microeddies $\left(d_{e}\right)$ is dependent on the solution viscosity $\eta$ and density $\rho$, as well as L. It can be estimated from Equation 17:

$$
d_{e}=\frac{1}{\rho}\left(\frac{\eta^{3} m}{N_{p} \omega^{3} L^{5}}\right)^{1 / 4}
$$

Where $m$ is the total mass of the solution in the reactor, and $N_{\mathrm{p}}$ is the power number. The latter term is indicative of the power consumed by the impeller. It is characteristic of the experimental setup and accounts for the type and position of the impeller, the design of the reactor, and the design features of the baffles if present. ${ }^{222}$ In the preparation of UF MCs, an exponential relationship between the average $\mathrm{MC}$ diameter and the shear rate was reported by Brown et al. (Figure 20). ${ }^{30,112}$

By studying the encapsulation of $n$-octadecane with a MF shell, Zhang et al. proposed Equation 18 to account for the increase in the capsule weight-average diameter or number-average diameter $(\mathrm{d})$ with the stirring rate $(\omega)::^{223}$

$$
d=d_{0}+A_{1} \mathrm{e}^{-\frac{\omega}{\omega_{1}}}
$$


The parameters $d_{0}, A_{1}$, and $\omega_{1}$ are fitting constants characteristic of the system. In the reported study, Equation 19 was found for the number-average diameter $\left(d_{n}\right)$ :

$$
d_{n}=0.94+334.49 \mathrm{e}^{-\frac{\omega}{1018.72}}
$$

In order to gain greater control over the size of UF microcapsules, Nesterova et al. compared four different stirrer geometries. ${ }^{224}$ The four-bladed metal propeller (Figure 21a) yielded linseed oil@UF capsules $154 \mu \mathrm{m}$ in diameter at a stirring rate of $1200 \mathrm{rpm}$. Greater stirring rates resulted in the shearing of the capsules, and the formation of a larger number of nano-sized materials. A gentler stirring was obtained with the three-bar glass stirrer (Figure 21b) resulting in larger capsule sizes. The intense stirring produced with the glass disc (Figure 21c) damaged the capsule walls. Somewhat larger capsule sizes were formed with the commonly used three-blade metal propeller (Figure 21d).

4 Proposed mechanisms for capsule formation

Details regarding the mechanism of MC formation from amino resins are still lacking, and even the classification of the microencapsulation process remains unclear. ${ }^{36}$ Various authors have postulated a process occurring strictly by in situ polymerization, by formation of a coacervation phase, or by their combination. The addition of a surfactant or additives may furthermore promote interfacial interactions, and thus implicate an interfacial polymerization process (Section 3.8.5).

As illustrated in Figure 2, Sliwka proposed a general description of the microencapsulation process in the case of complex coacervation for a mixture of gelatin and gum arabic according to four steps. ${ }^{12}$ The core material was dispersed in the continuous phase, followed by precipitation of the coacervate in the continuous phase. The micro-coacervate phase gradually precipitated at the 
surface of the core material, and eventually coalesced as a macro-coacervate phase to form the MC wall.

Dietrich et al. proposed a comparable and more detailed mechanism for the in situ polymerization of MF polymers over a hydrophobic core (methylparathion). ${ }^{166,225}$ The process was divided into three stages. ${ }^{200,226}$ In the first stage, a precondensate solution composed of oligomeric species was obtained by reacting melamine and formaldehyde under basic conditions at $80{ }^{\circ} \mathrm{C}$. After addition of the core material to the aqueous prepolymer solution, dispersion of the oil phase was promoted by the surface activity of the amino species, and the interfacial tension between the continuous and dispersed phases. In the second stage, the polycondensation of the oligomeric species was induced by lowering the $\mathrm{pH}$ of the solution. The increased concentration of the encapsulant at the interface, favored by hydrophobic/hydrophilic and electrostatic interactions with the core material, increased the rate of polycondensation at the boundary relative to the rate in the continuous phase. This effect favored the formation of a well-defined core-shell structure. During the last stage of the process, strengthening of the capsule wall occurred by further polymer deposition and cross-linking, and was accompanied by a reduction in the capsule size. The recovered capsules often displayed a granular surface. The surface activity of the MF condensate was found to be enhanced when reacted with methanol or triethanolamine, and promoted MC formation. UF prepolymers displayed less pronounced surfactant properties rendering the encapsulation process more difficult.

Salaün et al. reported a similar mechanism for the encapsulation of $n$-hexadecane with a methanol-functionalized melamine-formaldehyde (MMF) resin in presence of an added surfactant. $^{23,181}$ By monitoring the surface tension during the microencapsulation process, the authors proposed a mechanism involving the inception of a polymer-rich liquid phase in the first 
stage, reminiscent of a coacervate phase (also proposed by other authors). ${ }^{195,226}$ At the end of the reaction, the MCs displayed a rough surface which originated from the deposition and crosslinking of MF NPs. Fei et al. studied the encapsulation of a fragrance oil with a MMF resin in presence of poly(styrene-co-maleic anhydride) as a surfactant. The authors proposed a comparable mechanism, but they highlighted the change in particle size during the encapsulation process (Figure 22). ${ }^{31}$ The surfactant was found to stabilize the dispersion of the oil phase while promoting the adsorption of the polymer resin at the surface by electrostatic interactions. After deposition of the prepolymer, however, a redispersion of the droplet was observed as evidenced by the rapid decrease in particle size. Several intermediate morphologies were noted during the strengthening phase at $60{ }^{\circ} \mathrm{C}$ including non-adsorbed prepolymer, and vesicles. By further increasing the temperature to $75^{\circ} \mathrm{C}, \mathrm{MC}$ formation occurred with a concomitant decrease in particle size.

\section{Current interest in urea-formaldehyde microencapsulation}

\subsection{Trends in urea-formaldehyde microencapsulation}

The ability to contain materials in a micron-sized environment has proven to be a versatile and powerful technique applicable to numerous systems. This process allows the protection of a core material from its surroundings, or alternatively protection of the environment from an active core component. MCs also offer the ability to control the distribution and release of a core material at targeted sites. Numerous applications have benefited from the use of MCs including pressuresensitive recording devices, ${ }^{39,227}$ adhesive materials, ${ }^{52}$ agrochemicals, ${ }^{49}$ pharmaceuticals, ${ }^{18}$ phasechange materials, ${ }^{228}$ and electronic inks. ${ }^{154,158,229}$ Current interest in the use of amino resin MCs lies in the design of functional materials, with tunable and stimuli-responsive properties. Although amino resins are not well-suited for stimuli-responsive behavior on their own, stimuli-responsive 
materials can be incorporated in the core and wall of the microcapsule to provide stimuliresponsive properties. The concept was demonstrated for instance by $\mathrm{Chu}$ and co-workers by incorporating PNIPAM-co-PAAm sub-microspheres in the shell wall of chitosan microcapsules. $^{230}$ Other examples include decoration of the capsule-wall with micelles, ${ }^{231}$ magnetic nanoparticles, ${ }^{206}$ or using an electrophoretic fluid as core for displays. ${ }^{158}$ Materials with self-healing properties, ${ }^{53}$ tunable pore-size, ${ }^{230}$ and controlled-release properties ${ }^{232}$ are all examples of current research interests with promising applications in smart technologies.

\subsection{Self-healing materials}

Materials that can self-repair have attracted enormous interest in recent years, and are expected to play important roles in many engineering applications. ${ }^{233}$ Self-healing materials can help reduce replacement cost, increase material performance, and improve safety. A seminal report by White et al. demonstrated the use of UF MCs containing dicyclopentadiene dispersed in an epoxy matrix containing Grubbs' catalyst (Figure 23). ${ }^{53}$ The core material exuded when the capsules were under stress, due to induced crack formation, and polymerized when in contact with the catalyst. Relative to the damaged material, a significant improvement in the mechanical integrity of the material was obtained after healing.

\subsection{Multi-walled microcapsules}

MCs with varying wall composition have been prepared by relying on two different encapsulation processes, namely interfacial and in situ polymerization. Multi-walled MCs were obtained by using either a two-step or a one-step procedure. In the two-step approach, polyurethane capsules were first prepared by interfacial polymerization between a diisocyanate and a polyamine 
compound. ${ }^{234}$ The recovered MCs were subsequently used as a core for the deposition of a PUF wall by in situ polymerization. A one-step procedure was also reported by Caruso et al. involving the incorporation of a polyurethane prepolymer with isocyanate groups into the core material (ethyl phenylacetate). ${ }^{117}$ Dispersion of the binary core material in an aqueous solution of urea with further addition of formaldehyde yielded PU/UF MCs with a wall thickness ranging from $200 \mathrm{~nm}$ to 675 nm (Figure 24). MCs with a high thermal stability were thus obtained.

\subsection{Binary microcapsules}

MCs have been used as Pickering stabilizers in the preparation of larger MCs. For instance, UF MCs encapsulating dibutylphthalate and having diameters approximating $1.4 \mu \mathrm{m}$ acted as stabilizers for the dispersion of dicyclopentadiene. The latter compound was used as a core material and was subsequently encapsulated by interfacial polymerization of an isocyanate and a polyol (Figure 25). ${ }^{235}$ This strategy allowed the preparation of MCs containing two different cores. Such capsules could prove useful in self-healing material applications for instance.

\subsection{Porous microcapsules}

Nonionic surfactants can self-assemble into micelles above their cloud points. For example, upon an increase in temperature, dehydration of polyoxyethylene nonyl phenyl ether (NP-10) resulted in aggregation and micelle formation. When used during the encapsulation process, it was shown that these micelles could deposit at the surface of a core material, and migrate to the MC surface during the UF wall formation. Dissociation of the micelles upon lowering of the

temperature resulted in pore formation on the MCs surface (Figure 26). ${ }^{231}$ The pore size could be controlled by adjusting the temperature, and ranged from 5 to $200 \mathrm{~nm}$ in diameter. Porous MCs 
displaying a large specific surface area, and adjustable permeability could prove useful for the controlled release of a core material in applications such as drug or catalyst delivery.

\subsection{Cell encapsulation}

Major efforts have been made by researchers to develop encapsulation systems capable of a controlled and sustained delivery of a core material. Such systems may be of central importance in applications such as drug or pesticide delivery. Conventional single-walled MCs often do not provide sufficient control over the release of a core material, and are limited by a high initial burst release. Cells from microorganisms represent an attractive encapsulation system, and have been used in the encapsulation of various compounds (pharmaceuticals, anti-oxidants, essential oil, etc.). Zhang et al. demonstrated that algae cells are effective for the encapsulation of the pesticide tebuconazole (Figure 27) ${ }^{236}$ Further modification of the negatively-charged cell membrane with UF prepolymers resulted in MCs displaying controlled core-release and a reduced burst release effect. The efficacy of these materials was demonstrated in the sustained protection of wheat.

\section{Conclusions}

Microencapsulation is a versatile process that has allowed for the rational design of a wide range of advanced and functional materials. The protection of an active core material for targeted release, or the isolation of reactive compounds from the environment into micrometer-sized domains has spawned numerous applications. Examples of encapsulated materials include pharmaceuticals, agrochemicals, inks, oils, fragrances, perfumes and adhesives. Amino resin polymers display unique properties, such as high mechanical strength, good thermal stability, water and chemical resistance, low permeability, and low cost, making them attractive as wall- 
forming materials. The encapsulation process using urea and/or melamine and formaldehyde relies on the complex chemistry of amino resins to form a polymer network. Both the composition and operating conditions employed during the preparation of MCs are important considerations. Chemical, physical, and physiochemical properties of the system all affect the MC formation and the resulting material properties. Although amino resin MCs have been extensively employed for more than 50 years, the complexity of the process renders the selection of the experimental parameters challenging, and thus it has often remained an empirical process. Recently, a renewed interest in the field has led to a number of investigations probing the influence of various parameters, and has allowed for a more detailed description of the encapsulation process. Mechanistic models have emerged describing microencapsulation by in situ polymerization of amino resins. A complete understanding, however, is still lacking, and further research is needed to predict accurately the outcome of the encapsulation process. New characterization methods, as well as advances in polymer chemistry, colloidal science, nanotechnology, and engineering are expected to help bring greater understanding to this exciting field. Furthermore, promising avenues of research are expected to yield increasingly functional and smart materials combining a range of properties. Applications such as phase change-materials, self-healing structures, electronic inks, thermo-sensitive paper, smart coatings, and drug-delivery vehicles are just a few examples that illustrate the versatility and scope of the microencapsulation technology.

\section{References and footnotes}

\footnotetext{
* Also referred to as fill, payload, encapsulate, nucleus, internal phase, active ingredient, or active agent.

${ }^{\phi}$ Also known as shell, shell wall, coating, external phase or membrane.
} 
$\varphi$ Microencapsulation techniques are sometimes divided among three categories, viz. chemical, physico-chemical, and physico-mechanical methods. ${ }^{239,240}$

${ }^{\sigma}$ In situ polymerization in some instances is regarded as a type of interfacial polymerization.

1. Green, B. K. "Pressure sensitive record material" US Patent 2,712,507, 1955.

2. Green, B. K.; Schleicher, L. "Manifold record material" US Patent 2,730,456, 1956.

3. Green, B. K.; Schleicher, L. "Oil-containing microscopic capsules and method of making them" US Patent 2,800,457, 1957.

4. Cui, J.; Hao, J. "Nanoengineered polymer capsules: From fabrication to applications", In Self-Assembled Structures: Properties and Applications in Solution and on Surfaces; Hao, J., Ed.; CRC Press/Taylor and Francis: Boca Raton, 2011; p 236.

5. Arán-Ais, F.; Pérez-Limiñana, M. Á.; Sánchez-Navarro, M. M.; Orgilés-Barceló, C. "Developments in microencapsulation technology to improve adhesive formulations", J. Adhes. 2012, 88, 391-405.

6. Arshady, R.; George, M. H. "Suspension, dispersion, and interfacial polycondensation: A methodological survey", Polym. Eng. Sci. 1993, 33, 865-876.

7. Bansode, S. S.; Banarjee, S. K.; Gaikwad, D. D.; Jadhav, S. L.; Thorat, R. M. "Microencapsulation: A review", Int. J. Pharm. Sci. Rev. Res. 2010, 1, 38-43.

8. Gouin, S. "Microencapsulation: Industrial appraisal of existing technologies and trends", Trends Food Sci. Technol. 2004, 15, 330-347.

9. Jyothi, N. V. N.; Prasanna, P. M.; Sakarkar, S. N.; Prabha, K. S.; Ramaiah, P. S.; Srawan, G. Y. "Microencapsulation techniques, factors influencing encapsulation efficiency.", J. Microencapsulation 2010, 27, 187-197. 
10. Thies, C. "Microencapsulation", In Encyclopedia of Polymer Science and Technology; John Wiley and Sons, 2004; pp 1-29.

11. Shukla, P. G. "Microencapsulation of liquid active agents", In Functional Coatings; Ghosh, S. K., Ed.; Wiley-VCH: Weinheim, 2006; pp 153-186.

12. Sliwka, W. "Microencapsulation", Angew. Chemie Int. Ed. 1975, 14, 539-550.

13. Thies, C. "Physicochemical aspects of microencapsulation", Polym. Plast. Technol. Eng. $1975,5,1-22$.

14. Umer, H.; Nigam, H.; Tamboli, A. M.; Nainar, M. S. M. "Microencapsulation : Process, techniques and applications", Int. J. Res. Pharm. Biomed. Sci. 2011, 2, 474-481.

15. Thies, C. "Microencapsulation", In Van Nostrand's Encyclopedia of Chemistry; 2005; pp $1-6$.

16. Poncelet, D. "Microencapsulation: fundamentals, methods and applications", In Surface Chemistry in Biomedical and Environmental Science; Blitz, J. P., Gun'ko, V. M., Eds.; Springer: Dordrecht, 2006; pp 23-34.

17. Dubey, R.; Shami, T. C.; Bhasker Rao, K. U. "Microencapsulation technology and applications", Def. Sci. J. 2009, 59, 82-95.

18. "Microencapsulation: Methods and industrial applications"; Simon, B., Ed.; Marcel Dekker: New York, 1996.

19. "Functional coatings"; Ghosh, S. K., Ed.; Wiley-VCH: Weinheim, 2006.

20. "Microencapsulation: Processes and applications"; Vandegaer, J. E., Ed.; Plenum Press: New York, 1973. 
21. Mishra, M. K. "Overview of encapsulation and controlled release", In Handbook of Encapsulation and Controlled Release; Mishra, M., Ed.; Taylor and Francis: Boca Raton, 2016; pp 3-19.

22. Lamprecht, A.; Bodmeier, R. "Microencapsulation", In Ullmann's Encyclopedia of Industrial Chemistry; Wiley-VCH, 2010; pp 157-172.

23. Salaün, F. "Microencapsulation by interfacial polymerization", In Encapsulation Nanotechnologies; Mittal, V., Ed.; Scrivener Publishing/Wiley: Salem, MA, 2013; pp $137-173$.

24. Finch, C. A. "Industrial microencapsulation: polymers for microcapsule walls", In Encapsulation and Controlled Release; Karsa, D. R., Stephenson, R. A., Eds.; Woodhead Publishing Ltd: Abington, 1993; pp 1-12.

25. Marteaux, L. "Si-based inorganic microencapsulation", In Microencapsulation: Innovative Applications; Giamberini, M., Fernandez Prieto, S., Tylkowski, B., Eds.; de Gruyter: Berlin, 2015; pp 187-216.

26. Sun, G.; Zhang, Z. "Mechanical properties of melamine-formaldehyde microcapsules", J. Microencapsulation 2001, 18, 593-602.

27. Yuan, L.; Liang, G. Z.; Xie, J. Q.; Guo, J.; Li, L. "Thermal stability of microencapsulated epoxy resins with poly(urea-formaldehyde)", Polym. Degrad. Stab. 2006, 91, 2300-2306.

28. Powell, M. P. "Water-resistant micro-capsular opacifier system and products" US Patent $4,089,834,1978$.

29. Yuan, L.; Liang, G. Z.; Xie, J. Q.; Li, L.; Guo, J. "The permeability and stability of microencapsulated epoxy resins", J. Mater. Sci. 2007, 42, 4390-4397. 
30. Yuan, L.; Liang, G.; Xie, J.; Li, L.; Guo, J. "Preparation and characterization of poly(urea-formaldehyde) microcapsules filled with epoxy resins", Polymer 2006, 47, $5338-5349$.

31. Fei, X.; Zhao, H.; Zhang, B.; Cao, L.; Yu, M.; Zhou, J.; Yu, L. "Microencapsulation mechanism and size control of fragrance microcapsules with melamine resin shell", Colloids Surf. A 2015, 469, 300-306.

32. Liu, X.; Sheng, X.; Lee, J. K.; Kessler, M. R. "Synthesis and characterization of melamine- urea-formaldehyde microcapsules containing ENB-based self-healing agents", Macromol. Mater. Eng. 2009, 294, 389-395.

33. Duan, B. "Microencapsulation via in situ polymerization", In Handbook of Encapsulation and Controlled Release; Mishra, M., Ed.; Taylor and Francis: Boca Raton, 2016; pp 307314.

34. Boh, B.; Sumiga, B. "In situ polymerisation microcapsules", Bioencapsulation Innov. $2013,4-6$.

35. Dietrich, K.; Herma, H.; Nastke, R.; Bonatz, E.; Teige, W. "Amino resin microcapsules. I. Literature and patent review", Acta Polym. 1989, 40, 243-251.

36. Sobel, R.; Versic, R.; Gaonkar, A. G. "Introduction to Microencapsulation and Controlled Delivery in Foods"; Gaonkar, A. G., Vasisht, N., Khare, A. R., Sobel, R., Eds.; Elsevier Inc., 2014.

37. Thies, C. "Physicochemical aspects of microencapsulation", Polym. Plast. Technol. Eng. $1975,5,1-22$.

38. Percy, S. R. "Improvement in drying and concentrating liquid substances by atomizing" US Patent 125,406, 1872. 
39. Macaulay, N. "Recording paper coated with microscopic capsules of coloring material, capsules and method of making" US Patent 3,016,308, 1962.

40. Bungenberg de Jong, H. G.; Kruyt, H. R. "Koacervation", Kolloid Zeitschrift 1930, 50, $39-48$.

41. Thies, C. "A survey of microencapsulation processes", In Microencapsulation - Methods and Industrial Applications; Simon, B., Ed.; Marcel Dekker: New York, 1996; pp 1-19.

42. Vassiliades, A. E. "Microcapsules. process for their formation and transfer sheet record material coated therewith" US Patent 3,993,831, 1976.

43. Hasler, D. J.; McGhee, T. A. "Microcapsules methods for their preparation, and sheet material carrying microcapsules" US Patent 4,105,823, 1978.

44. Zhang, Y.; Rochefort, D. "Characterisation and applications of microcapsules obtained by interfacial polycondensation", J. Microencapsulation 2012, 29, 1-14.

45. Baxter, G. "Microencapsulation processes in modern business forms", In Microencapsulation: Processes and Applications; Vandegaer, J. E., Ed.; Plenum Press: New York, 1974; pp 127-143.

46. Veatch, F.; Burhans, R. W. "Process of producing hollow particles and resulting product" US Patent 2,797,201, 1957.

47. Soloway, S. "Encapsulation of natural products" US Patent 3,137,631, 1964.

48. Geary, R. J. "Particulate pesticidal composition coated with an amido-aldehyde resin polymerized in situ" US Patent 3,074,845, 1963.

49. Matson, G. W. "Microcapsules and process of making" US Patent 3,516,941, 1970.

50. Matson, G. W. "Microcapsules-containing paper" US Patent 3,516,846, 1970. 
51. Shrewsbury, R. W. "Copy sheet having a layer of thermally rupturable hollow microcapsules on a conductive backing and the method of use" US Patent 3,318,697, 1967.

52. Deckert, F. W.; Matson, G. W. "Sealant having epoxide encapsulated by aminoplast shell and polymeric binder" US Patent 3,642,937, 1972.

53. White, S. R.; Sottos, N. R.; Geubelle, P. H.; Moore, J. S.; Kessler, M. R.; Sriram, S. R.; Brown, E. N.; Viswanathan, S. "Autonomic healing of polymer composites", Nature 2001, 409, 794-797.

54. Shchukin, D.; Möhwald, H. "A coat of many applications", Science 2013, 341, 14581459.

55. Tong, X.-M.; Zhang, T.; Yang, M.-Z.; Zhang, Q. "Preparation and characterization of novel melamine modified poly(urea-formaldehyde) self-repairing microcapsules", Colloids Surf., A 2010, 371, 91-97.

56. Wang, R.; Hu, H.; Liu, W.; He, X.; Guo, Q. "The Effect of synthesis condition on physical properties of epoxy-containing microcapsules", J. Appl. Polym. Sci. 2012, 124, $1866-1879$.

57. Yin, T.; Rong, M. Z.; Zhang, M. Q.; Yang, G. C. "Self-healing epoxy composites Preparation and effect of the healant consisting of microencapsulated epoxy and latent curing agent", Compos. Sci. Technol. 2007, 67, 201-212.

58. Cosco, S.; Ambrogi, V.; Musto, P.; Carfagna, C. "Urea-formaldehyde microcapsules containing an epoxy resin: Influence of reaction parameters on the encapsulation yield", Macromol. Symp. 2006, 234, 184-192. 
59. Cosco, S.; Ambrogi, V.; Musto, P.; Carfagna, C. "Properties of poly(urea-formaldehyde) microcapsules containing an epoxy resin", J. Appl. Polym. Sci. 2007, 105, 1400-1411.

60. Blaiszik, B. J.; Caruso, M. M.; McIlroy, D. A.; Moore, J. S.; White, S. R.; Sottos, N. R. "Microcapsules filled with reactive solutions for self-healing materials", Polymer 2009, 50, 990-997.

61. Diem, H.; Matthias, G.; Wagner, R. A. "Amino resins", In Ullmann's Encyclopedia of Industrial Chemistry; WILEY-VCH Verlag, 2012; pp 79-106.

62. Braun, D.; Ritzert, H.-J. "Urea-formaldehyde and melamine-formaldehyde polymers", In Comprehensive Polymer Science and Supplements; Allen, G., Bevington, J. C., Eds.; Pergamon Press: Oxford, 1989; pp 649-665.

63. Brydson, J. A. "Aminoplastics". In Plastics Materials; Butterworth-Heinemann: Oxford, 1999; pp 668-693.

64. Dunky, M. "Urea-formaldehyde (UF) adhesive resins for wood", Int. J. Adhes. Adhes. $1998,18,95-107$.

65. Pizzi, A. "Urea-formaldehyde adhesives". In Handbook of Adhesive Technology; Pizzi, A., Mittal, K. L., Eds.; Dekker: New York, 2003.

66. Williams, L. L. "Amino resins and plastics", In Encyclopedia of polymer science and technology; John Wiley \& Sons, 2003; Vol. 1, pp 340-371.

67. Crowe, G. A.; Lynch, C. L. "Urea-formaldehyde kinetic studies", J. Am. Chem. Soc. 1948, 70, 3795-3797.

68. Crowe, G. A.; Lynch, C. C. "Polarographic urea-formaldehyde kinetic studies", J. Am. Chem. Soc. 1949, 71, 3731-3733. 
69. Smets, G.; Borzee, A. "Réactions de condensation de formaldéhyde et d'urée", J. Polym. Sci. $1952,8,371-394$.

70. de Jong, J. I. "Note on the reaction of urea and formaldehyde", Recl. des Trav. Chim. des Pays-Bas 1950, 69, 1566.

71. de Jong, J. I.; de Jonge, J. "The reaction of urea with formaldehyde", Recl. des Trav. Chim. des Pays-Bas 1952, 71, 643-660.

72. de Jong, J. I.; de Jonge, J. "The formation and decomposition of dimethylolurea", Recl. des Trav. Chim. des Pays-Bas 1952, 71, 661-667.

73. de Jong, J. I.; de Jonge, J.; Eden, H. A. K. "The formation of trimethylol urea.", Recl. des Trav. Chim. des Pays-Bas 1953, 72, 88-90.

74. de Jong, J. I.; de Jonge, J. "Kinetics of the formation of methylene linkages in solutions of urea and formaldehyde", Recl. des Trav. Chim. des Pays-Bas 1953, 72, 139-156.

75. de Jong, J. I.; de Jong, J. "The hydrolyis of methylene diurea", Recl. des Trav. Chim. des Pays-Bas 1953, 72, 202-206.

76. de Jong, J. I.; de Jonge, J. "Kinetics of the reaction between mono- methylolurea and methylene diurea", Recl. des Trav. Chim. des Pays-Bas 1953, 72, $207-212$.

77. Landqvist, N. "On the reaction between urea and formaldehyde in neutral and alkaline solutions .1. Experimental studies of the rates of the equimolecular reaction", Acta Chem. Scand. 1955, 9, 1127-1142.

78. Landqvist, N. "On the reaction between urea and formaldehyde in neutral and alkaline solutions .2. Experimental studies of the rates of the equimolecular reaction between monomethylol urea and formaldehyde", Acta Chem. Scand. 1955, 9, 1459-1465. 
79. Landqvist, N. "On the Reaction between urea and formaldehyde in neutral and alkaline solutions .3. Experimental studies of the rates of hydrolysis of monomethylol urea", Acta Chem. Scand. 1955, 9, 1466-1470.

80. Landqvist, N. "On the reaction between urea and formaldehyde in neutral and alkaline solutions .4. Experimental Studies of the Rates of hydrolysis of dimethylol urea", Acta Chem. Scand. 1955, 9, 1471-1476.

81. Landqvist, N. "On the reaction between urea and formaldehyde in neutral and alkaline solutions .5. Experimental studies of the order of the "equimolecular" reaction between urea and formaldehyde at equal concentrations of the reactants", Acta Chem. Scand. $1955,9,1477-1483$.

82. Landqvist, N. "On the reaction between urea and formaldehyde in neutral and alkaline solutions .6. Experimental Studies of the activation energy and the heat of reaction", Acta Chem. Scand. 1956, 10, 244-248.

83. Landqvist, N. "On the reaction between urea and formaldehyde in neutral and alkaline solutions .7. A spectrophotometrical method for quantitative determination of urea and methylol ureas of the reaction mixture", Acta Chem. Scand. 1957, 11, 776-779.

84. Landqvist, N. "On the reaction between urea and formaldehyde in neutral and alkaline solutions .8. Studies of the reactions and equilibria at formaldehyde-urea molecular ratios in the range 1.4-2.0", Acta Chem. Scand. 1957, 11, 780-785.

85. Landqvist, N. "On the reaction between urea and formaldehyde in neutral and alkaline solutions .9. The influence of methanol on the rates of reaction", Acta Chem. Scand. 1957, 11, 786-791. 
86. Landqvist, N. "On the reaction between urea and formaldehyde in neutral and alkaline solutions. 10. Notes on the reaction mechanism", Acta Chem. Scand. 1957, 11, 792-803.

87. Steinhof, O.; Kibrik, É. J.; Scherr, G.; Hasse, H. "Quantitative and qualitative ${ }^{1} \mathrm{H},{ }^{13} \mathrm{C}$, and ${ }^{15} \mathrm{~N}$ NMR spectroscopic investigation of the urea-formaldehyde resin synthesis.", Magn. Reson. Chem. 2014, 52, 138-162.

88. Winkelman, J. G. M.; Voorwinde, O. K.; Ottens, M.; Beenackers, A. A. C. M.; Janssen, L. P. B. M. "Kinetics and chemical equilibrium of the hydration of formaldehyde", Chem. Eng. Sci. 2002, 57, 4067-4076.

89. Walker, J. F. "Formaldehyde"; Reinhold Publishing Corp.: New York, 1944.

90. Gaca, K. Z.; Parkinson, J. A.; Lue, L.; Sefcik, J. "Equilibrium speciation in moderately concentrated formaldehyde - methanol - water solutions investigated using ${ }^{13} \mathrm{C}$ and ${ }^{1} \mathrm{H}$ nuclear magnetic resonance spectroscopy", Ind. Eng. Chem. Res. 2014, 53, 9262-9271.

91. Wadano, M. "Über die saure Natur wäßriger Formaldehyd-Lösungen.", Berichte der Dtsch. Chem. Gesellschaft 1934, 67, 191-197.

92. Gaál, F. F.; Avramović, B. D. "Determination of total free acid in formaldehyde by coulometric catalytic thermometric titration", J. Therm. Anal. 1983, 26, 285-289.

93. Bowman, P. I.; Burton, J. "Stabilization of the acidity of formaldehyde solutions" US Patent 2,253,999, 1941.

94. Ott, M.; Fischer, H. H.; Maiwald, M.; Albert, K.; Hasse, H. "Kinetics of oligomerization reactions in formaldehyde solutions: NMR experiments up to $373 \mathrm{~K}$ and thermodynamically consistent model", Chem. Eng. Process. Process Intensif. 2005, 44, $653-660$. 
95. Glutz, B. R.; Zollinger, H. "Allgemeine Säure-Basen-Katalyse der MonomethylolBildung aus Harnstoff und Formaldehyd in Wasser", Helv. Chim. Acta 1976, 52, 19761984.

96. Nair, B. R.; Francis, D. J. "Kinetics and mechanism of urea- formaldehyde reaction", Polymer 1983, 24, 626-630.

97. Lei, H.; Frazier, C. E. "Curing behavior of melamine-urea-formaldehyde (MUF) resin adhesive", Int. J. Adhes. Adhes. 2015, 62, 40-44.

98. Glutz, B. R.; Zollinger, H. "Allgemeine Säure-Basen-Katalyse der MonomethylolBildung aus Harnstoff und Formaldehyd in Wasser", Helv. Chim. Acta 1969, 52, 19761984.

99. Li, T. H.; Wang, C. M.; Xie, X. G.; Du, G. Ben. "A computational exploration of the mechanisms for the acid-catalytic urea-formaldehyde reaction: new insight into the old topic", J. Phys. Org. Chem. 2012, 25, 118-125.

100. Chuang, I.-S.; Maciel, G. E. " ${ }^{13}$ C CP/MAS NMR study of the structural dependence of urea-formaldehyde resins on formaldehyde-to-urea molar ratios at different urea concentrations and pH values", Macromolecules 1992, 25, 3204-3226.

101. Ferra, J. M. M.; Mendes, A. M.; Costa, M. R. N.; Carvalho, L. H.; Magalhães, F. D. "A study on the colloidal nature of urea-formaldehyde resins and its relation with adhesive performance", J. Appl. Polym. Sci. 2010, 118, 1956-1968.

102. Despres, A.; Pizzi, A. "Colloidal aggregation of aminoplastic polycondensation resins: Urea-formaldehyde versus melamine-formaldehyde and melamine-urea-formaldehyde resins", J. Appl. Polym. Sci. 2006, 100, 1406-1412. 
103. Dunker, A. K.; John, W. E.; Rammon, R.; Farmer, B.; Johns, S. J. "Slightly bizarre protein chemistry: Urea-formaldehyde resin from a biochemical perspective", J. Adhes. 1986, 19, 153-176.

104. Motter, W. K. "The formation of the colloidal phase in low mole ratio urea-formaldehyde resins", Washington State University, 1990.

105. Pratt, T. J.; Johns, W. E.; Rammon, R. M.; Plagemann, W. L. "A novel concept on the structure of cured urea-formaldehyde resin", J. Adhes. 1985, 17, 275-295.

106. Mehdiabadi, S.; Nehzat, M. S.; Bagheri, R. "Correlating viscosity in urea-formaldehyde polymerization", J. Appl. Polym. Sci. 1998, 69, 631-636.

107. Gray, A.; Egan, S.; Bakalis, S.; Zhang, Z. "Determination of microcapsule physicochemical, structural, and mechanical properties", Particuology 2016, 24, 32-43.

108. Bao, L.; Zhang, F.; Chen, T.; Cao, J.; Chen, Y.; Bai, Y. "Novel submicron poly(ureaformaldehyde) and essence of jasmine microcapsules with enhanced sustained release", Flavour Fragr. J. 2015, 30, 459-466.

109. Silva, A. F. T.; Burggraeve, A.; Denon, Q.; Van Der Meeren, P.; Sandler, N.; Van Den Kerkhof, T.; Hellings, M.; Vervaet, C.; Remon, J. P.; Lopes, J. A.; De Beer, T. "Particle sizing measurements in pharmaceutical applications: Comparison of in-process methods versus off-line methods", Eur. J. Pharm. Biopharm. 2013, 85, 1006-1018.

110. Treffer, D.; Wahl, P. R.; Hörmann, T. R.; Markl, D.; Schrank, S.; Jones, I.; Cruise, P.; Mürb, R. K.; Koscher, G.; Roblegg, E.; Khinast, J. G. "In-line implementation of an image-based particle size measurement tool to monitor hot-melt extruded pellets", Int. J. Pharm. 2014, 466, 181-189. 
111. Chuanjie, F.; Juntao, T.; Xiaodong, Z. "Effects of process parameters on the physical properties of poly (urea-formaldehyde) microcapsules prepared by a one-step method", Iran. Polym. J. (English Ed.) 2013, 22, 665-675.

112. Brown, E. N.; Kessler, M. R.; Sottos, N. R.; White, S. R. "In situ poly(ureaformaldehyde) microencapsulation of dicyclopentadiene.", J. Microencapsulation 2003, 20, 719-730.

113. Pretzl, M.; Neubauer, M.; Tekaat, M.; Kunert, C.; Kuttner, C.; Leon, G.; Berthier, D.; Erni, P.; Ouali, L.; Fery, A. "Formation and mechanical characterization of aminoplast core/ shell microcapsules", Appl. Mater. Interfaces 2012, 4, 2940-2948.

114. Ambrosi, M.; Fratini, E.; Baglioni, P.; Vannucci, C.; Bartolini, A.; Pintens, A.; Smets, J. "Microcapsules for confining fluids: Prediction of shell stability from advanced SAXS investigations", J. Phys. Chem. C 2016, 120, 13514-13522.

115. Yuan, L.; Chen, F.; Gu, A.; Liang, G.; Lin, C.; Huang, S.; Nutt, S.; Chen, G.; Gao, Y. "Synthesis of poly(urea-formaldehyde) encapsulated dibutyltin dilaurate through the selfcatalysis of core materials", Polym. Bull. 2014, 71, 261-273.

116. Lebedeva, O. V.; Kim, B. S.; Vinogradova, O. I. "Mechanical properties of polyelectrolyte-filled multilayer microcapsules studied by atomic force and confocal microscopy", Langmuir 2004, 20, 10685-10690.

117. Caruso, M. M.; Blaiszik, B. J.; Jin, H.; Schelkopf, S. R.; Stradley, D. S.; Sottos, N. R.; White, S. R.; Moore, J. S. "Robust, double-walled microcapsules for self-healing polymeric materials", ACS Appl. Mater. Interfaces 2010, 2, 1195-1199.

118. Dubreuil, F.; Elsner, N.; Fery, A. "Elastic properties of polyelectrolyte capsules studied by atomic-force microscopy and RICM", Eur. Phys. J. E 2003, 12, 215-221. 
119. Adi, S.; Adi, H.; Chan, H. K.; Young, P. M.; Traini, D.; Yang, R.; Yu, A. "Scanning white-light interferometry as a novel technique to quantify the surface roughness of micron-sized particles for inhalation", Langmuir 2008, 24, 11307-11312.

120. Xie, H. G.; Zheng, J. N.; Li, X. X.; Liu, X. D.; Zhu, J.; Wang, F.; Xie, W. Y.; Ma, X. J. "Effect of surface morphology and charge on the amount and conformation of fibrinogen adsorbed onto alginate/chitosan microcapsules", Langmuir 2010, 26, 5587-5594.

121. Kietzke, T.; Stiller, B.; Landfester, K.; Montenegro, R.; Neher, D. "Probing the local optical properties of layers prepared from polymer nanoparticles", Synth. Met. 2005, 152, $101-104$.

122. Utama, R. H.; Dulle, M.; Förster, S.; Stenzel, M. H.; Zetterlund, P. B. "SAXS analysis of shell formation during nanocapsule synthesis via inverse miniemulsion periphery RAFT polymerization", Macromol. Rapid Commun. 2015, 36, 1267-1271.

123. Lv, L.; Yang, Z.; Chen, G.; Zhu, G.; Han, N.; Schlangen, E.; Xing, F. "Synthesis and characterization of a new polymeric microcapsule and feasibility investigation in selfhealing cementitious materials", Constr. Build. Mater. 2016, 105, 487-495.

124. Barigou, M. "Particle tracking in opaque mixing systems: An overview of the capabilities of PET and PEPT", Chem. Eng. Res. Des. 2004, 82, 1258-1267.

125. Zaleski, R.; Maciejewska, M.; Puzio, M. "Mechanical stability of porous copolymers by positron annihilation lifetime spectroscopy", J. Phys. Chem. C 2015, 119, 11636-11645.

126. Wang, Y.; Bai, W. Y.; Cui, Z. C. "Fourier transform infrared spectroscopic study of pesticide microcapsules influenced by formaldehyde to urea ratio", Spectrosc. Lett. 2015, 48, 259-264. 
127. Suryanarayana, C.; Rao, K. C.; Kumar, D. "Preparation and characterization of microcapsules containing linseed oil and its use in self-healing coatings", Prog. Org. Coatings 2008, 63, 72-78.

128. Zeng, F.; Polanuyer, B. "Method for measuring encapsulation efficiency for hydrophobic actives" US Patent 9,575,036, 2017.

129. Li, H.; Wang, R.; Hu, H.; Liu, W. "Surface modification of self-healing poly(ureaformaldehyde) microcapsules using silane-coupling agent", Appl. Surf. Sci. 2008, 255, 1894-1900.

130. Nguyen, L.-T. T.; Hillewaere, X. K. D.; Teixeira, R. F. a.; van den Berg, O.; Du Prez, F. E. "Efficient microencapsulation of a liquid isocyanate with in situ shell functionalization", Polym. Chem. 2015, 6, 1159-1170.

131. Caruso, M. M.; Blaiszik, B. J.; White, S. R.; Sottos, N. R.; Moore, J. S. "Full recovery of fracture toughness using a nontoxic solvent-based self-healing system", Adv. Funct. Mater. 2008, 18, 1898-1904.

132. Fan, C.; Tang, J.; Zhou, X. "Role of ammonium chloride in preparing poly(ureaformaldehyde) microcapsules using one-step method", J. Appl. Polym. Sci. 2013, 129, $2848-2856$.

133. Shi, H.; Liu, F.; Han, E. H. "Surface-engineered microcapsules by layer-by-layer assembling for entrapment of corrosion inhibitor", J. Mater. Sci. Technol. 2015, 31, 512516.

134. Su, J.; Wang, L.; Ren, L. "Fabrication and thermal properties of microPCMs: Used melamine-formaldehyde resin as shell material", J. Appl. Polym. Sci. 2006, 101, 15221528. 
135. Li, B.; Dong, G.; Zhang, C. "Storage stability and solubility of poly(urea- formaldehyde) microcapsules containing a-olefin drag reducing polymer", J. Appl. Polym. Sci. 2011, 122, 1450-1456.

136. Zhang, X. X.; Tao, X. M.; Yick, K. L.; Fan, Y. F. "Expansion space and thermal stability of microencapsulated n-octadecane", J. Appl. Polym. Sci. 2005, 97, 390-396.

137. Wang, R.; Li, H.; Hu, H.; He, X.; Liu, W. "Preparation and characterization of selfhealing microcapsules with poly(urea-formaldehyde) grafted epoxy functional group shell", J. Appl. Polym. Sci. 2009, 113, 1501-1506.

138. Neubauer, M. P.; Poehlmann, M.; Fery, A. "Microcapsule mechanics: From stability to function", Adv. Colloid Interface Sci. 2014, 207, 65-80.

139. Mercadé-Prieto, R.; Allen, R.; Zhang, Z.; York, D.; Preece, J. A.; Goodwin, T. E. "Failure of elastic-plastic core-shell microcapsules under compression", AIChE J. 2012, $58,2674-2681$.

140. Erkan, G.; Sariişik, M.; Pazarlioğlu, N. K. "The microencapsulation of terbinafine via in situ polymerization of melamine-formaldehyde and their application to cotton fabric", Polym. Appl. Polym. Sci. 2010, 118, 3707-3714.

141. Feng, C.; Zhang, C.; Kong, F.; Wang, J. "Synthesis of thiodiazole copper microcapsules and release behavior of inhibiting R. solanacearum", RSC Adv. 2014, 4, 4478-4486.

142. Noh, H. H.; Lee, J. K. "Microencapsulation of self-healing agents containing a fluorescent dye", Express Polym. Lett. 2013, 7, 88-94.

143. Mercadé-Prieto, R.; Pan, X.; Fernández-González, A.; Zhang, Z.; Bakalis, S. "Quantification of microcapsules deposited in cotton fabrics before and after abrasion using fluorescence microscopy", Ind. Eng. Chem. Res. 2012, 51, 16741-16749. 
144. Long, Y.; Vincent, B.; York, D.; Zhang, Z.; Preece, J. A. "Organic-inorganic double shell composite microcapsules", Chem. Commun. 2010, 46, 1718.

145. Igari, Y.; Hori, Y.; Okamoto, T. "Microcapsule and process for production thereof" US Patent 6,486,099, 2002.

146. Sansukcharearnpon, A.; Wanichwecharungruang, S.; Leepipatpaiboon, N.; Kerdcharoen, T.; Arayachukeat, S. "High loading fragrance encapsulation based on a polymer-blend: Preparation and release behavior", Int. J. Pharm. 2010, 391, 267-273.

147. Zhang, D. X.; Li, B. X.; Zhang, X. P.; Zhang, Z. Q.; Wang, W. C.; Liu, F. "Phoxim microcapsules prepared with polyurea and urea-formaldehyde resins differ in photostability and insecticidal activity", J. Agric. Food Chem. 2016, 64, 2841-2846.

148. Kage, H.; Kawahara, H.; Hamada, N.; Kotake, T.; Ogura, H. "Operating conditions and microcapsules generated by in situ polymerization", Adv. Powder Technol. 2002, 13, $265-285$.

149. Charbonneau, J. W.; Law, R. S.; Ludwig, W. J.; Otteson, W. O. "Pressure-sensitive making materials" US Patent 4,201,404, 1980.

150. Park, S.-J.; Shin, Y.-S.; Lee, J.-R. "Preparation and characterization of microcapsules containing lemon oil", J. Colloid Interface Sci. 2001, 241, 502-508.

151. Petrovic, L. B.; Sovilj, V. J.; Katona, J. M.; Milanovic, J. L. "Influence of polymersurfactant interactions on o/w emulsion properties and microcapsule formation", J. Colloid Interface Sci. 2010, 342, 333-339.

152. Wu, K.; Wang, Z.; Hu, Y. "Microencapsulated ammonium polyphosphate with ureamelamine-formaldehyde shell: preparation, characterization, and its flame retardance in polypropylene", Polym. Adv. Technol. 2008, 19, 1118-1128. 
153. Shin, Y.; Yoo, D. I.; Son, K. "Development of thermoregulating textile materials with microencapsulated phase change materials (PCM). II. Preparation and application of PCM microcapsules", J. Appl. Polym. Sci. 2005, 96, 2005-2010.

154. Wang, Y. T.; Zhao, X. P.; Wang, D. W. "Electrophoretic ink using urea-formaldehyde microspheres.", J. Microencapsulation 2006, 23, 762-768.

155. Morishita, S.; Matsushita, T. "Heat-sensitive recording sheet" US Patent 4,520,376, 1985.

156. Yuan, L.; Gu, A.; Liang, G. "Preparation and properties of poly(urea-formaldehyde) microcapsules filled with epoxy resins", Mater. Chem. Phys. 2008, 110, 417-425.

157. Marathe, R. J.; Chaudhari, A. B.; Hedaoo, R. K.; Sohn, D.; Chaudhari, V. R.; Gite, V. V. "Urea formaldehyde (UF) microcapsules loaded with corrosion inhibitor for enhancing the anti-corrosive properties of acrylic-based multifunctional PU coatings", RSC Adv. $2015,5,15539-15546$.

158. Li, X. X.; Zhang, B.; Feng, Y. Q.; Li, X. G.; Wen, Z. Q. "Synthesis and characterisation of urea-formaldehyde microcapsules containing electrophoretic fluid with red-white particles", Mater. Res. Innov. 2012, 16, 261-266.

159. Qin, R.; Xu, G.; Guo, L.; Jiang, Y.; Ding, R. "Preparation and characterization of a novel poly(urea-formaldehyde) microcapsules with similar reflectance spectrum to leaves in the UV-Vis-NIR region of 300-2500 nm", Mater. Chem. Phys. 2012, 136, 737-743.

160. Fan, C.; Zhou, X. "Influence of operating conditions on the surface morphology of microcapsules prepared by in situ polymerization", Colloids Surfaces A Physicochem. Eng. Asp. 2010, 363, 49-55.

161. Hildebrand, J. H.; Scott, R. L. "The Solubility of Nonelectrolytes"; Reinhold: New York, 1950. 
162. Hansen, C. M. "Hansen solubility parameters: A user's handbook"; Hansen, C. M., Ed.; CRC Press: Boca Raton, 2000.

163. Stöver, H. D. H.; Li, W.-H.; Croll, L. M. "Method of encapsulating hydrophobic organic molecules in polyurea capsules" US Patent 0,271,735, 2005.

164. Hofmeister, I.; Landfester, K.; Taden, A. "Controlled formation of polymer nanocapsules with high diffusion-barrier properties and prediction of encapsulation efficiency", Angew. Chem. Int. Ed. 2015, 54, 327-330.

165. Latnikova, A.; Grigoriev, D.; Möhwald, H.; Shchukin, D. "Microgel containers for selfhealing polymeric materials: Morphology prediction and mechanism of formation", Polymer 2015, 73, 183-194.

166. Dietrich, K.; Bonatz, E.; Nastke, R.; Herma, H.; Walter, M.; Teige, W. "Amino resin microcapsules iv. Surface tension of the resins and mechanism of capsule formation", Acta Polym. 1990, 41, 91-94.

167. Fan, C.; Zhou, X. "Effect of emulsifier on poly(urea-formaldehyde) microencapsulation of tetrachloroethylene", Polym. Bull. 2011, 67, 15-27.

168. Tang, J.; Fan, C.; Lin, Q.; Zhou, X. "Smooth, stable and optically transparent microcapsules prepared by one-step method using sodium carboxymethyl cellulose as protective colloid", Colloids Surf., A 2014, 459, 65-73.

169. Chuanjie, F.; Xiaodong, Z. "Preparation and barrier properties of the microcapsules added nanoclays in the wall", Polym. Adv. Technol. 2009, 20, 934-939.

170. Lee, H. Y.; Lee, S. J.; Cheong, I. W.; Kim, J. H. "Microencapsulation of fragrant oil via in situ polymerization: Effects of $\mathrm{pH}$ and melamine-formaldehyde molar ratio.", J. Microencapsulation 2002, 19, 559-569. 
171. Sgraja, M.; Blömer, J.; Bertling, J.; Jansens, P. J. "In situ encapsulation of tetradecene droplets in oil-in-water emulsions using amino resins", J. Appl. Polym. Sci. 2008, 110, 2366-2373.

172. Rochmadi, A. P.; Hasokowati, W. "Mechanism of microencapsulation with ureaformaldehyde polymer", Am. J. Appl. Sci. 2010, 7, 739-745.

173. Hwang, J.-S.; Kim, J.-N.; Wee, Y.-J.; Jang, H.-G.; Kim, S.-H.; Ryu, H.-W. "Factors affecting the characteristics of melamine resin microcapsules containing frangran oils", Biotechnol. Bioprocess Eng. 2006, 11, 391-395.

174. Sanghvi, S. P.; Nairn, J. G. "Effect of viscosity and interfacial tension on particle size of cellulose acetate trimellitate microspheres.", J. Microencapsulation 1992, 9, 215-227.

175. Wang, J.; Zhao, X.; Guo, H.; Zheng, Q. "Preparation of microcapsules containing twophase core materials", Langmuir 2004, 20, 10845-10850.

176. Landfester, K.; Bechthold, N.; Tiarks, F.; Antonietti, M. "Formulation and stability mechanisms of polymerizable miniemulsions", Macromolecules 1999, 32, 5222-5228.

177. Torza, S.; Mason, S. G. "Three-phase interactions in shear and electrical fields", J. Colloid Interface Sci. 1970, 33, 67-83.

178. Van Oss, C. J. "Polymer surfaces and interfaces II"; Feast, W. J., Munro, H. S., Richards, R. W., Eds.; Wiley: New York, 1993.

179. Thomasin, C.; Nam-Trân, H.; Merkle, H. P.; Gander, B. "Drug microencapsulation by PLA/PLGA coacervation in the light of thermodynamics. 1. Overview and theoretical considerations", J. Pharm. Sci. 1998, 87, 259-268.

180. Guo, H. L.; Zhao, X. P.; Wang, J. P. "The relation between narrow-dispersed microcapsules and surfactants.", J. Microencapsulation 2005, 22, 853-862. 
181. Salaün, F.; Devaux, E.; Bourbigot, S.; Rumeau, P. "Influence of process parameters on microcapsules loaded with $n$-hexadecane prepared by in situ polymerization", Chem. Eng. J. 2009, 155, 457-465.

182. Griffin, W. C. "Classification of surface-active agents by "HLB”", J. Soc. Cosmet. Chem. $1949,1,311-326$.

183. Griffin, W. C. "Calculation of HLB values of non-ionic surfactants", J. Soc. Cosmet. Chem. 1954, 5, 249-256.

184. Davies, J. T. "A quantitative kinetic theory of emulsion type. I. Physical chemistry of the emulsifying agent". In 2nd International Congress Surface Activitiy; Butterworths, London, 1957; pp 426-438.

185. ICI Americas. "The HLB System: A time saving guide to emulsifier selection"; Wilmington, 1980.

186. Pasquali, R. C.; Taurozzi, M. P.; Bregni, C. "Some considerations about the hydrophiliclipophilic balance system", Int. J. Pharm. 2008, 356, 44-51.

187. Golden, R. "Process for production of encapsulated water-dispersible materials" US Patent 4,157,983, 1973.

188. Yin, D.; Ma, L.; Geng, W.; Zhang, B.; Zhang, Q. "Microencapsulation of $n$-hexadecanol by in situ polymerization of melamine-formaldehyde resin in emulsion stabilized by styrene--maleic anhydride copolymer", Int. J. Energy Res. 2015, 39, 661-667.

189. Holtzscherer, C.; Candau, F. "Application of the cohesive energy ratio concept (CER) to the formation of polymerizable microemulsions", Colloids and Surfaces 1988, 29, 411423. 
190. Little, R. C. "Correlation of surfactant hydrophile-lipophile balance (HLB) with solubility parameter", J. Colloid Interface Sci. 1978, 65, 587-588.

191. Beerbower, A.; Hill, M. W. "Application of cohesive energy ratio (CER) concept to anionic surfactants", Am. Cosmet. Perfum 1972, 87, 85.

192. Winsor, P. A. "Solvent properties of amphiphilic compounds"; Butterworths: London, 1954.

193. Hoshi, Y.; Matsukawa, H. "Process for preparing microcapsules by polymerization of urea and formaldehyde in the presence of gum arabic" US Patent 4,221,710, 1980.

194. Foris, P. L.; Brown, R. W.; Phillips, P. S. J. "Capsule manufacture" US Patent 4,001,140, 1977.

195. Foris, P. L.; Brown, R. W.; Phillips, P. S. J. "Capsule manufacture "US Patent 4,100,103, 1978.

196. Fuchigami, M. "Micro-capsules and method for their production" US Patent 4,223,178, 1980.

197. Saeki, K.; Matsukawa, H.; Satomura, M. "Method for preparing microcapsules" US Patent 4,251,386, 1981.

198. Hoshi, Y.; Matsukawa, H. "Process for the production of microcapsules" US Patent $4,353,809,1982$.

199. Schibler, L. "Process for the encapsulation of dispersible materials" US Patent 3,594,328, 1971.

200. Yoshizawa, H.; Kamio, E.; Hirabayashi, N.; Jacobson, J.; Kitamura, Y. "Membrane formation mechanism of cross-linked polyurea microcapsules by phase separation method", J. Microencapsulation 2004, 21, 241-249. 
201. Yoshizawa, H.; Kamio, E.; Kobayashi, E.; Jacobson, J.; Kitamura, Y. "Investigation of alternative compounds to poly(E-MA) as a polymeric surfactant for preparation of microcapsules by phase separation method.", J. Microencapsulation 2007, 24, 349-357.

202. Fereidoon, A.; Ghorbanzadeh Ahangari, M.; Jahanshahi, M. "Effect of nanoparticles on the morphology and thermal properties of self-healing poly(urea-formaldehyde) microcapsules", J. Polym. Res. 2013, 20.

203. Song, Q.; Li, Y.; Xing, J.; Hu, J. Y.; Marcus, Y. "Thermal stability of composite phase change material microcapsules incorporated with silver nano-particles", Polymer 2007, 48, 3317-3323.

204. Niu, X.-W.; Sun, Y.-M.; Ding, S.-N.; Chen, C.-C.; Song, B.; Xu, H.-B.; Qi, Z.-J.; Qi, Q. "Synthesis of enhanced urea-formaldehyde resin microcapsules doped with nanotitania", J. Appl. Polym. Sci. 2011, 124, 248-256.

205. Ghorbanzadeh Ahangari, M.; Fereidoon, A.; Jahanshahi, M.; Sharifi, N. "Effect of nanoparticles on the micromechanical and surface properties of poly(urea-formaldehyde) composite microcapsules", Compos. Part B Eng. 2014, 56, 450-455.

206. Huang, Y.; Xuan, Y. M.; Li, Q.; Che, J. F. "Preparation and characterization of magnetic phase-change microcapsules", Chinese Sci. Bull. 2009, 54, 318-323.

207. Hayford, D. E. "Capsule manufacture" US Patent 4,444,699, 1984.

208. Richmond, H. H.; Myers, G. S.; Wright, G. F. "The reaction between formaldehyde and ammonia", J. Am. Chem. Soc. 1948, 70, 3659-3664.

209. Scopelitis, E.; Pizzi, A. "Urea-resorcinol-formaldehyde adhesives of low resorcinol content", J. Appl. Polym. Sci. 1993, 48, 2135-2146. 
210. Huang, Z.; Yu, X.; Li, W.; Liu, S. "Preparation of urea-formaldehyde paraffin microcapsules modified by carboxymethyl cellulose as a potential phase change material", J. For. Res. 2015, 26, 253-260.

211. Blaiszik, B. J.; Sottos, N. R.; White, S. R. "Nanocapsules for self-healing materials", Compos. Sci. Technol. 2008, 68, 978-986.

212. IARC. "Monographs on the evaluation of carcinogenic risk to humans"; Lyon, 2006; Vol. 88.

213. Smets, J.; Dihora, J. O.; Pintens, A.; Guinebretiere, S. J.; Druckrey, A. K.; Sands, P. D.; Yan, N. "Benefit agent containing delivery particle" US Patent 8,551,935, 2013.

214. Boran, S.; Usta, M.; Gümüşkaya, E. "Decreasing formaldehyde emission from medium density fiberboard panels produced by adding different amine compounds to urea formaldehyde resin", Int. J. Adhes. Adhes. 2011, 31, 674-678.

215. Costa, N. A.; Pereira, J.; Ferra, J.; Cruz, P.; Martins, J.; Magalhaes, F. D.; Mendes, A.; Carvalho, L. H. "Scavengers for achieving zero formaldehyde emission of wood-based panels", Wood Sci. Technol. 2013, 47, 1261-1272.

216. Braeckman, K. G.; Depoot, K. J. M.; Van Pachtenbeke, T. R. M.; Smets, J. "Composition comprising microcapsules" US Patent 0,235,525, 2014.

217. Calabrese, R. V.; Chang, T. P. K.; Dang, P. T. "Drop breakup in turbulent stirred tank contactors. Part I: Effect of dispersed phase viscosity.", AIChE J. 1986, 32, 657-666.

218. Kolmogorov, A. N. "The Local structure of turbulence in incompressible viscous fluid for very large Reynolds numbers", Dokl. Akad. Nauk SSSR 1941, 30, 301.

219. Sprow, F. B. "Distribution of drop sizes produced in turbulent liquid—liquid dispersion", Chem. Eng. Sci. 1967, 22, 435-442. 
220. Armenante, P. M.; Kirwan, D. J. "Mass transfer to microparticles in agitated systems", Chem. Eng. Sci. 1989, 44, 2781-2796.

221. Dobetti, L.; Pantaleo, V. "Application of a hydrodynamic model to microencapsulation by coacervation.", J. Microencapsulation 2002, 19, 139-151.

222. Furukawa, H.; Kato, Y.; Inoue, Y.; Kato, T.; Tada, Y.; Hashimoto, S. "Correlation of power consumption for several kinds of mixing impellers", Int. J. Chem. Eng. 2012, 2012, 106496.

223. Zhang, X. X.; Fan, Y. F.; Tao, X. M.; Yick, K. L. "Fabrication and properties of microcapsules and nanocapsules containing n-octadecane", Mater. Chem. Phys. 2004, 88, $300-307$.

224. Nesterova, T.; Dam-Johansen, K.; Pedersen, L. T.; Kiil, S. "Microcapsule-based selfhealing anticorrosive coatings: Capsule size, coating formulation, and exposure testing", Prog. Org. Coatings 2012, 75, 309-318.

225. Dietrich, K.; Bonatz, E.; Geistlinger, H.; Herma, H.; Nastke, R.; Purz, H.-J.; Schlawne, M.; Teige, W. "Amino resin microcapsules. II. Preparation and morphology", Acta Polym. 1989, 40, 325-331.

226. Su, J.-F.; Huang, Z.; Ren, L. "High compact melamine-formaldehyde microPCMs containing n-octadecane fabricated by a two-step coacervation method", Colloid Polym. Sci. 2007, 285, 1581-1591.

227. Iwasaki, H.; Irii, S. "Microcapsules, method for their production and pressure sensitive copying sheet" US Patent 4,413,843, 1983. 
228. Zhao, C. Y.; Zhang, G. H. "Review on microencapsulated phase change materials (MEPCMs): Fabrication, characterization and applications", Renew. Sustain. Energy Rev. $2011,15,3813-3832$.

229. Song, J. K.; Choi, H. J.; Chin, I. "Preparation and properties of electrophoretic microcapsules for electronic paper.", J. Microencapsulation 2007, 24, 11-19.

230. Wei, J.; Ju, X. J.; Zou, X. Y.; Xie, R.; Wang, W.; Liu, Y. M.; Chu, L. Y. "Multi-stimuliresponsive microcapsules for adjustable controlled-release", Adv. Funct. Mater. 2014, 24, $3312-3323$.

231. Fu, D.; Su, Y.; Xie, B.; Liu, G.; Li, Z.; Jiang, K.; Wang, D. "Pore decoration on microcapsule surface using nonionic surfactant micelles as template: Temperature effect and encapsulation mechanism investigation", Colloids Surf., A 2011, 384, 219-227.

232. Kulkarni, A. R.; Soppimath, K. S.; Aminabhavi, T. M. "Urea-formaldehyde nanocapsules for the controlled release of diclofenac sodium.", J. Microencapsulation 2000, 17, 449458.

233. Blaiszik, B. J.; Kramer, S. L. B.; Olugebefola, S. C.; Moore, J. S.; Sottos, N. R.; White, S. R. "Self-healing polymers and composites", Annu. Rev. Mater. Res. 2010, 40, 179-211.

234. Li, G.; Feng, Y.; Gao, P.; Li, X. "Preparation of mono-dispersed polyurea-urea formaldehyde double layered microcapsules", Polym. Bull. 2008, 60, 725-731.

235. Mookhoek, S. D.; Blaiszik, B. J.; Fischer, H. R.; Sottos, N. R.; White, S. R.; Van Der Zwaag, S. "Peripherally decorated binary microcapsules containing two liquids", J. Mater. Chem. 2008, 18, 5390-5394. 
236. Zhang, B.; Zhang, T.; Wang, Q.; Ren, T. "Microorganism-based monodisperse microcapsules: encapsulation of the fungicide tebuconazole and its controlled release properties", RSC Adv. 2015, 5, 25164-25170.

237. Mark, J. E. "Physical Properties of Polymers Handbook", 2nd Ed.; Mark, J. E., Ed.; Springer Science and Business: New York, 2007.

238. Li, T. H.; Wang, C. M.; Xie, X. G.; Du, G. Ben. "A computational exploration of the mechanisms for the acid-catalytic urea-formaldehyde reaction: new insight into the old topic", J. Phys. Org. Chem. 2012, 25, 118-125.

239. Ghosh, S. K. "Functional coatings and microencapsulation: A general perspective", In Functional Coatings; Ghosh, S. K., Ed.; Wiley-VCH: Weinheim, 2006; pp 1-28.

240. Jyothi, N. V. N.; Prasanna, P. M.; Sakarkar, S. N.; Prabha, K. S.; Ramaiah, P. S.; Srawan, G. Y. "Microencapsulation techniques, factors influencing encapsulation efficiency", J. Microencapsulation 2010, 27, 187-197. 


\section{Captions}

Figure 1. Chemical structures of formaldehyde, urea, and melamine.

Figure 2. Illustration of the microencapsulation process via in situ polymerization.

Figure 3. Illustration of some important chemical encapsulation processes (not to scale). Coacervation process: (a) Dispersion of a core material in a homogeneous polymer solution; (b) Formation of a coacervate phase upon a change in solution conditions; (c) coating of the core with the coacervate phase, and onset of coalescence; (d) formation of the shell. Interfacial polymerization: (a') Dissolution of monomer A in the continuous phase, and monomer B in the dispersed phase; (b') diffusion of the monomers to the interface; (c') polymerization reaction between the monomers. In situ polymerization: (a") Dissolution of the monomers A and B in the continuous phase; (b") polymer formation; (c") precipitation of the polymer and deposition at the interface. Adapted with permission from Ref. 12. Copyright 1975 Wiley-VCH.

Figure 4. Number of academic articles and patents published per year. (From Orbit ${ }^{\mathrm{TM}}$ and Web of Science ${ }^{\mathrm{TM}}$, using combinations of the keywords: *capsul*, formaldehyde, urea, melamine, amino resin and aminoplast.)

Figure 5. General process for the synthesis of microcapsules by in situ polymerization.

Figure 6. Reaction rate constants as a function of $\mathrm{pH}$ for the addition and condensation reactions in urea-formaldehyde aqueous solutions at $35^{\circ} \mathrm{C}$. $\mathrm{U}=$ urea; $\mathrm{F}=$ formaldehyde; UF = urea- 
formaldehyde; $\mathrm{MMU}=$ monomethylol urea; $\mathrm{DMU}=$ dimethylol urea. The addition reaction rate constant $\mathrm{k}=0.56 \times 10^{-4}+0.14\left[\mathrm{H}^{+}\right]+1.7\left[\mathrm{HO}^{-}\right]$, and condensation reaction rate constant are obtained from de Jong and de Jonge. ${ }^{71,74}$

Figure 7. Schematic of the composition of urea-formaldehyde solutions during resin synthesis. Solution (a) before and (b) after flocculation by dilution. Adapted with permission from Ref. 101. Copyright 2010 Wiley.

Figure 8. (a) Evolution of the $\mathrm{pH}$, temperature, and capsule morphology during the microencapsulation of dicyclopentadiene with poly(urea-formaldehyde). (b) Formation of a binary surface composed of a smooth inner layer, and a rough outer surface. Reproduced with permission from Ref. 112. Copyright 2003 Taylor and Francis.

Figure 9. Evolution of the MF surface thickness with the wall material concentration $\left(c_{w}\right)$ over the total droplet surface area (A) during encapsulation of tetradecene. A critical capsule wall ca. 100 nm can be noted. Adapted with permission from Ref. 171. Copyright 2008 Wiley.

Figure 10. Equilibrium configurations taking place between two liquid phases (phase-1, and phase-3) dispersed in a third liquid medium (phase-2) for different sets of $S_{i}$ values. Adapted with permission from Ref. 177. Copyright 1970 Elsevier.

Figure 11. Surface and interfacial tension of melamine-formaldehyde resin. Reproduced with permission from Ref. 171. Copyright 2008 Wiley. 
Figure 12. Interfacial tension of amino resins composed of formaldehyde (FA), urea (U), melamine (M), and/or etherified methylol melamine (MeM). Ethanolamine (EA), diethanolamine (DEA), and triethanolamine were used as modifiers. Adapted with permission from Ref. 166. Copyright 1990 Akademie.

Figure 13. Evolution of the surface tension, melting enthalpy, and $\mathrm{pH}$ during the encapsulation reaction of $\mathrm{n}$-hexadecane with etherified melamine-formaldehyde resin. Reproduced with permission from Ref. 181. Copyright 2009 Elsevier.

Figure 14. Effect of the $\mathrm{pH}$ on the surface tension during the encapsulation of n-hexadecane with melamine-formaldehyde in presence of various amounts of surfactants (Tween-20 and Brij-35) in water. Reproduced with permission from Ref. 181. Copyright 2009 Elsevier.

Figure 15. Schematic illustration of the adsorption of surfactants at the core/wall interphase during microencapsulation. a) Poly(ethylene-alt-maleic anhydride) introduced reactive sites favorable to membrane formation, while b) SDS and c) Solsperse 17,000 hindered further deposition of poly(EMA). Adapted with permission from Ref. 200. Copyright 2004 Taylor and Francis.

Figure 16. Optical micrographs of microcapsules prepared using poly(ethylene-alt-maleic anhydride) in varying concentration: (a) $0.2 \mathrm{wt} \%$, (b) $0.4 \mathrm{wt} \%$, and (c) $0.67 \mathrm{wt} \%$. Reproduced with permission from Ref. 160. Copyright 2010 Elsevier. 
Figure 17. Proposed model for the poly(urea-formaldehyde) microcapsule formation in presence of titania nanoparticles. Reproduced with permission from Ref. 204. Copyright 2001 Wiley.

Figure 18. (a) Poly(urea-formaldehyde)-iron oxide nanoparticle microcapsules dispersed in water, and collected with a magnet on the side of a beaker. Reproduced with permission from Ref. ${ }^{206}$. Copyright Springer 2009 (c) Schematic of the effect of layered nanosilicates on the diffusion of a core material. Reproduced with permission from Ref. 169. Copyright Wiley 2008.

Figure 19. Effect of $\mathrm{NH}_{4} \mathrm{Cl}$ salt on the $\mathrm{pH}$ during the encapsulation of tetrachloroethylene with urea-formaldehyde. Reproduced with permission from Ref. 132. Copyright 2013 Wiley.

Figure 20. Variation of the average diameter of poly(urea-formaldehyde) microcapsules with the agitation rate. Reproduced with permission from Ref. 112. Copyright 2003 Taylor and Francis.

Figure 21. Different propellers used for the formation of poly(urea-formaldehyde) microcapsules: (a) pitched-blade metal turbine; (b) v-shaped glass propeller; (c) glass disc; (d) marine propeller. Reproduced with permission from Ref. 224. Copyright 2012 Elsevier.

Figure 22. Schematic illustration of the microencapsulation process by in situ polymerization. Reproduced with permission from Ref. 31. Copyright 2015 Elsevier B.V.

Figure 23. Self-healing material prepared by dispersing urea-formaldehyde microcapsules containing a healing agent: (a) The propagation of cracks in the matrix; (b) Rupture of the 
microcapsules; (c) Release of the healing agent that polymerizes upon interaction with a catalyst. Reproduced with permission from Ref. 53. Copyright 2001 Nature Publishing Group.

Figure 24. AFM phase image displaying the multi-wall composition of a microtomed poly(urethane)/poly(urea-formaldehyde) microcapsule prepared by a one-step process. Reproduced with permission from Ref. 117. Copyright 2010 American Chemical Society.

Figure 25. Binary microcapsule obtained by Pickering emulsion with UF microcapsules. Reprinted with permission from Ref. 235. Copyright 2008 The Royal Society of Chemistry.

Figure 26. Schematic illustration of the preparation of porous microcapsules by in situ polymerization of melamine-formaldehyde in presence of self-assembled surfactants (micelles), A-G. H. SEM images of the porous microcapsules obtained from this method. Reproduced with permission from Ref. 231. Copyright 2011 Elsevier B.V.

Figure 27. Illustration of the preparation of microorganism-based microcapsules by encapsulation of algae cells within poly(urea-formaldehyde) for the controlled release of a load material. Adapted with permission from Ref. 236. Copyright 2015 The Royal Society of Chemistry.

Table 1. Common methods used in microencapsulation processes.

Table 2. Important methods used in the characterization of microcapsules. Adapted from Ref. 107. 
Table 3. Techniques used for the characterization of the mechanical properties of microcapsules. Adapted from Ref. 107.

Table 4. Hansen solubility parameters, solubility sphere radius $\left(\mathrm{R}_{0}\right)$, solubility parameter distance $\left(\mathrm{R}_{\mathrm{a}}\right)$, and relative energy difference (RED) of representative compounds at $25^{\circ} \mathrm{C} .{ }^{237}$

Table 5. Order of importance (and optimal values) of the experimental conditions used in the encapsulation of epoxy resin in urea-formaldehyde microcapsules with respect to different determining parameters. Results obtained from orthogonal studies from Refs. 56,175 and 111.

Table 6. Suggested HLB values for surfactants used in various types of applications. ${ }^{183,185}$

Scheme 1. Condensation and alkoxylation reactions of formaldehyde in water-methanol mixtures. Formaldehyde is readily hydrated to methylene glycol in water $\left(\mathrm{K}_{\mathrm{h}}=1271\right.$ at $\left.25^{\circ} \mathrm{C}\right),{ }^{88}$ and further reactions form poly(methylene glycol)s. In presence of an alcohol, alkoxylated species are also formed.

Scheme 2. Equilibrium speciation for formaldehyde solutions in water-ethanol mixtures. Equilibrium constants were recalculated from the data reported by Gaca et al. ${ }^{90}$

Scheme 3. Overview of the reactions taking place during the polymerization of urea and formaldehyde, $\mathrm{ni} \geq 0$. Analogous reactions occur for melamine. 
Scheme 4. Proposed reaction mechanism and calculated relative energies for reaction intermediates involved in the acid-catalyzed reaction of urea and formaldehyde, computed by density functional theory by Li et al. Adapted from Ref. 238 .

Scheme 5. Reactions of ammonium chloride with formaldehyde in water. 
Table 1. Common methods used in microencapsulation processes.

\begin{tabular}{ll}
\hline Chemical methods (type A) & Physical/mechanical methods (type B) \\
\hline Interfacial polymerization & Spray drying \\
In situ polymerization & Multiple nozzle spraying \\
Polycondensation & Fluidized bed coating \\
Coacervation & Centrifugal extrusion \\
Phase separation & Extrusion \\
Layer-by-layer assembly & Microfluidic channels \\
\hline
\end{tabular}


Table 2. Important methods used in the characterization of microcapsules. Adapted from Ref. 107.

\begin{tabular}{|c|c|c|}
\hline Property & Method & Resolution \\
\hline \multirow{10}{*}{$\begin{array}{l}\text { Size, size } \\
\text { distribution }\end{array}$} & Dynamic light scattering (DLS) & $1 \mathrm{~nm}-1 \mu \mathrm{m}$ \\
\hline & & \\
\hline & Laser diffraction (LD) & $10 \mathrm{~nm}-3 \mathrm{~mm}$ \\
\hline & Scanning electron microscopy (SEM) & $>10 \mathrm{~nm}$ \\
\hline & Optical microscopy (OM) & $>400 \mathrm{~nm}$ \\
\hline & Chord length measurement: & \\
\hline & Focused beam reflectance measurement (FBRM) & $3 \mu \mathrm{m}-3 \mathrm{~mm}$ \\
\hline & Spatial filtering velocimetry (SFV) & $50 \mu \mathrm{m}-6 \mathrm{~mm}$ \\
\hline & Photometric stereo imaging & $>20 \mu \mathrm{m}$ \\
\hline & Sieve analysis & $>38 \mu \mathrm{m}$ \\
\hline \multirow[t]{3}{*}{ Surface roughness } & SEM & qualitative \\
\hline & Atomic force microscopy (AFM) & $<1 \mathrm{~nm}$ \\
\hline & Interferometry & $<1 \mathrm{~nm}$ \\
\hline Chemical & Fourier-transform infrared (FTIR) and Raman & $\mu \mathrm{m}$ \\
\hline \multirow[t]{4}{*}{ composition } & spectroscopies & \\
\hline & X-ray photoelectron spectroscopy (XPS) & $<100 \mathrm{~nm}$ \\
\hline & $\begin{array}{l}\text { Time-of-flight secondary-ion mass spectrometry } \\
\text { (TOF-SIMS) }\end{array}$ & $1-2 \mathrm{~nm}$ \\
\hline & Energy-dispersive X-ray spectroscopy (EDS) & $<2 \mu \mathrm{m}$ \\
\hline Zeta potential & Streaming potential & - \\
\hline
\end{tabular}


Electrophoresis

Wall thickness

Pore size

SEM, ESEM

AFM (PALS)
Focused ion beam (FIB) (coupled with SEM)

Transmission electron microscopy (TEM)

Confocal laser scanning microscopy (CLSM)

Small-angle X-ray scattering (SAXS)

X-ray computed tomography (CT)

Positron emission annihilation lifetime spectroscopy
$<30 \mu \mathrm{m}$

$>10 \mathrm{~nm}$

$>10 \mathrm{~nm}$

$0.2 \mathrm{~nm}$

$0.2-0.5 \mu \mathrm{m}$

$<200 \mathrm{~nm}$

$1-10 \mu \mathrm{m}$

$<1 \mathrm{~nm}$

$<30 \mathrm{~nm}$
Thermal properties
Crystallinity
Differential scanning calorimetry (DSC)
Thermo-gravimetric analysis (TGA)
Wide-angle X-ray diffraction (XRD) 
Table 3. Techniques used for the characterization of the mechanical properties of microcapsules. Adapted from Ref. 107.

\begin{tabular}{ll}
\hline Bulk characterization & Individual microcapsule \\
\hline Mechanical shaking & Optical tweezers \\
Turbine reactor & Shear flow \\
Compression & AFM \\
& Micromanipulte aspiration \\
\hline
\end{tabular}


Table 4. Hansen solubility parameters, solubility sphere radius $\left(\mathrm{R}_{0}\right)$, solubility parameter distance $\left(\mathrm{R}_{\mathrm{a}}\right)$, and relative energy difference (RED) of representative compounds at $25^{\circ} \mathrm{C} .{ }^{237}$

\begin{tabular}{|c|c|c|c|c|c|c|c|}
\hline \multirow[b]{2}{*}{ Compound } & \multicolumn{6}{|c|}{$\left[(\mathrm{MPa})^{1 / 2}\right]$} & \multirow[b]{2}{*}{$R E D^{a}$} \\
\hline & $\delta$ & $\delta_{d}$ & $\delta_{p}$ & $\bar{\delta} \delta_{h}$ & $R_{0}$ & $R_{a}^{a}$ & \\
\hline $\begin{array}{l}\text { Urea-formaldehyde resin } \\
\text { (Plastopal H, BASF) }^{162}\end{array}$ & 26.9 & 20.8 & 8.3 & 15.0 & 12.7 & - & - \\
\hline Epoxy resin (Epikote 1001, Shell) ${ }^{162}$ & 26.3 & 20.4 & 12.0 & 11.5 & 12.7 & 5.2 & 0.4 \\
\hline Epoxy resin (Epon 1001) $)^{162}$ & 23.2 & 18.1 & 11.4 & 9.0 & 9.1 & 8.6 & 0.7 \\
\hline Acetone & 20.1 & 15.5 & 10.4 & 7.0 & - & 13.4 & 1.1 \\
\hline Benzene & 18.6 & 18.4 & 0.0 & 2.0 & - & 16.2 & 1.3 \\
\hline Chloroform & 19.0 & 17.8 & 3.1 & 5.7 & - & 12.2 & 1.0 \\
\hline Diethyl ether & 15.8 & 14.5 & 2.9 & 5.1 & - & 16.9 & 1.3 \\
\hline Dioxane-1,4 & 20.5 & 19.0 & 1.8 & 7.4 & - & 10.6 & 0.8 \\
\hline Ethanol & 26.0 & 15.8 & 8.8 & 19.4 & - & 10.9 & 0.9 \\
\hline Styrene & 19.0 & 18.6 & 1.0 & 4.1 & - & 13.8 & 1.1 \\
\hline Tetrahydrofuran & 19.4 & 16.8 & 5.7 & 8.0 & - & 10.9 & 0.9 \\
\hline Toluene & 18.2 & 18.0 & 1.4 & 2.0 & - & 15.7 & 1.2 \\
\hline Water & 47.9 & 15.5 & 16.0 & 42.4 & - & 30.4 & 2.4 \\
\hline Xylene $(o)$ & 18.0 & 17.8 & 1.0 & 3.1 & - & 15.2 & 1.2 \\
\hline
\end{tabular}

\footnotetext{
a. Values relative to urea-formaldehyde resin.
} 
Table 5. Order of importance (and optimal values) of the experimental conditions used in the encapsulation of epoxy resin in urea-formaldehyde microcapsules with respect to different determining parameters. Results obtained from orthogonal studies from Refs. 56,175 and 111.

\begin{tabular}{lllll}
\hline Determining parameter & Variable 1 & Variable 2 & Variable 3 & Variable 4 \\
\hline Viscosity & Time & Urea/FA mole ratio & Temp. & Initial $\mathrm{pH}$ \\
& $(1 \mathrm{~h})$ & $(1: 1.75)$ & $\left(75^{\circ} \mathrm{C}\right)$ & $(9)$ \\
Yield & Emulsifier & Time & Core/shell & Stirring rate \\
& SDBS $(1.5 \mathrm{wt} \%)$ & $(3 \mathrm{~h})$ & $(1.4: 1)$ & $(250 \mathrm{rpm})$ \\
Core content & Core/shell & Time & Stirring rate & \\
Diameter & Stirring rate & Time & Core/shell & \\
Shell thickness & Stirring rate & Core $/ \mathrm{shell}^{2}$ & Time & Emulsifier \\
Yield & Initial $\mathrm{pH}$ & Heating rate & Wall conc. & $\mathrm{NaCl}$ \\
& $(\mathrm{pH} 3.5)$ & $\left(0.5^{\circ} \mathrm{C} \cdot \mathrm{min}^{-1}\right)$ & $\left(36 \mathrm{~g} \cdot \mathrm{L}^{-1}\right)$ & $\left(50 \mathrm{~g} \cdot \mathrm{L}^{-1}\right)$ \\
\hline
\end{tabular}


Table 6. Suggested HLB values for surfactants used in various types of applications. ${ }^{183,185}$

\begin{tabular}{lc}
\hline Type of application & HLB Values \\
\hline Water-in-oil emulsifier & $4-6$ \\
Wetting agent & $7-9$ \\
Oil-in-water emulsifier & $8-18$ \\
Detergent & $13-15$ \\
Solubilizer & $10-18$ \\
\hline
\end{tabular}

